\title{
Diseño y desarrollo de un aplicativo móvil educativo para optimizar la comunicación e interacción entre los miembros de las instituciones educativas en tiempo real
}

\author{
MARÍA Elena RuIZ RIVERA ${ }^{1}$ \\ GeORGE TORRES DÁvila ${ }^{2}$ \\ EDGAR RUIZ LIZAMA ${ }^{3}$
}

ReCibido: 15/01/2021 Aceptado: 26/05/2021 Publicado: 26/07/2021

\begin{abstract}
RESUMEN
El objetivo de este trabajo es optimizar la comunicación e interacción entre los miembros de las instituciones educativas, mediante una aplicación móvil que permita mejorar el nivel de servicio de la institución. Al utilizar la base de datos con la información recolectada por la institución, como fuente importante para la integración y la recopilación de información en el sector educativo, se considera cada institución como una entidad, entonces se podría afirmar que todas las personas que interactúan con ella son la población, la cual necesita estar conectada y comunicada entre sí para subsistir. Bajo las premisas expuestas, se diseña una aplicación que tiene como finalidad reunir en una sola plataforma a los usuarios que interactúan en cada institución, de tal manera que se mantengan comunicados y conectados entre sí al definir roles específicos, los cuales tendrán habilitadas funciones específicas para cada tipo de usuario.
\end{abstract}

Palabras clave: aplicación móvil; sistema móvil; Cloud; Cloud Messaging Firebase.

\section{INTRODUCCIÓN}

La idea de crear esta aplicación móvil surge de la intención de elevar el nivel de la comunicación e interacción entre los miembros de una institución educativa para que estén cada vez más cerca y así logren interactuar de la mejor manera en beneficio propio y de la institución en la que se encuentran.

Una de las motivaciones para realizar este trabajo fue el deseo de impulsar el rendimiento académico de los estudiantes mediante la mejor de procesos básicos, pero fundamentales para las instituciones educativas como son la comunicación y la interacción entre sus integrantes. Si se lograra acercar a las personas involucradas en el desarrollo académico de cada estudiante a tal punto que logren avanzar hacia objetivos académicos, las probabilidades para que el desarrollo académico sea el esperado y el más óptimo en pro de la educación del alumno serían mayores, puesto que la interacción sería personalizada y cada vez más cercana.

Para proponer una posible solución a la problemática expuesta, se realizaron encuestas a los estudiantes, padres y profesores de instituciones educativas de nivel inicial, primaria y secundaria. Las respuestas obtenidas revelaron los aspectos que deben ser mejorados, desde la comunicación de tareas, pasando por la atención personalizada de dudas, hasta un registro de asistencias y notas, el cual se pueda consultar en cualquier momento; asimismo, se determinó que muchas veces no se les notifica a los padres cuando hay una tarea o se ha recibido una nota, usualmente, porque los alumnos no se las dan a sus padres, lo que genera una brecha entre alumnos, padres y profesores.

1 Licenciada en Computación. Docente de la Facultad de Ingeniería de Sistemas e Informática, Universidad Nacional Mayor de San Marcos. (Lima, Perú).

ORCID: https://orcid.org/0000-0003-3300-7068

Autor de correspondencia: mruizr@unmsm.edu.pe

2 Egresado de la Facultad de Ingeniería de Sistemas e Informática, Universidad Nacional Mayor de San Marcos. Analista en Inteligencia de Negocios. (Lima, Perú).

ORCID: https://orcid.org/0000-0003-0086-5066

E-mail: george.deave.95@gmail.com

3 Magíster en Informática por la Pontificia Universidad Católica del Perú. Docente de la Facultad de Ingeniería Industrial, Universidad Nacional Mayor de San Marcos. (Lima, Perú). ORCID: https://orcid.org/0000-0001-9403-1358

E-mail: eruizl@unmsm.edu.pe 
Por todo esto, se propone una aplicación móvil que se adapte a las necesidades de la institución educativa, que reduzca al mínimo esa brecha y, a su vez, optimice la comunicación e interacción. Otro punto importante es que estará destinada también a padres y profesores que, en su mayoría, son ajenos a las nuevas tecnologías (Muñoz et al., 2015), por ello se propone una aplicación móvil de interfaz amigable y fácil uso. La propuesta intenta acortar la brecha entre las personas pertenecientes a cada institución, mediante el uso intuitivo y fácil de una aplicación móvil que tiene como principales pilares a la comunicación y la interacción (Paredes-Parada, 2019).

Esta aplicación móvil se debe de diferenciar de las herramientas que se usan en la actualidad, tales como las aplicaciones de mensajerías, en las cuales las asignaciones y/o tareas se pierden entre tantos mensajes o todo se pone en una única sala de chat (Herrera y Buenabad, 2013). Se deben proporcionar las herramientas necesarias para que la educación sea la óptima, y ayudar en las actividades más básicas para lograr una mejora en la etapa de aprendizaje de los estudiantes (Gértrudix y Barroso, 2016).

Por naturaleza, el ser humano es un ser sociable que necesita saber, investigar, informarse, tal es así que la comunicación ha sufrido varios cambios en métodos, tipos y metodologías; estudios a gran escala demuestran que tanto la información como la comunicación son vitales para poder lograr cambios notables en la vida del ser humano (Molina et al., 2015). La comunicación ya no solo se realiza de forma verbal, oral o escrita, pues ahora existe la tecnología que le permite al ser humano tener una conectividad e interconectividad visualizando imágenes y escuchando sonidos en tiempo real, lo que es aplicable a escala global (Cataldi et al., 2012). En los últimos años, la interconectividad entre personas de todo el mundo ha ido en aumento en casi todos los sectores de la población y en todos los niveles socioculturales, gracias al uso de la tecnología y al manejo correcto de herramientas como los dispositivos móviles "smartphones", que día a día son adquiridos por millones de personas y utilizados mayormente para la comunicación e información a nivel mundial gracias al internet (Gomez et al, 2016).

En este proyecto de investigación se busca optimizar la comunicación e interacción entre los miembros de las instituciones educativas, es decir, hacer más fluida la relación profesor o tutor - padre de familia, director - profesores, y más, con la utilización de los dispositivos móviles. La integración en las instituciones educativas mediante el uso de dispositivos móviles para la enseñanza y aprendizaje, está tomando fuerza. Villalonga y Marta-Lazo (2015) mencionan que para llevar con éxito cada etapa de su modelo de aprendizaje se deben usar ciertas herramientas ya consolidadas como Google Drive, Facebook, Twitter, etc.; las autoras afirman que dichas herramientas deben utilizarse por separado para cada módulo del modelo de aprendizaje, esto ahora debe cambiar ya que esas funcionalidades se pueden incluir en una sola app y cumplir las funciones que ellos realizan por separado. Al tener datos estadísticos reales tras realizar encuestas y contar con una data ordenada, se definieron dos roles: el de docentes y el de alumnos. Ambos grupos fueron entrevistados para conocer la realidad de las actividades educativas, y los resultados obtenidos fueron alentadores; se realizaron preguntas acerca de su tipo de dispositivo móvil, la compañía contratada, las aplicaciones utilizadas con más frecuencia, el costo de la red, la frecuencia de uso del teléfono, etc. (Mendoza, 2014). Estos datos son muy útiles para cualquier tipo de investigación referente a educación moderna, puesto que permiten observar cómo se van formando las bases para afrontar una nueva etapa en la educación de una manera más tecnológica y sistematizada (Cataldi et al, 2012). El uso de los dispositivos móviles como una herramienta que sirva de mediador entre la enseñanza y el aprendizaje debe verse como la oportunidad de otorgarle un valor agregado a la educación (Mendoza, 2014). Este trabajo se enfoca en la utilización de los dispositivos móviles para optimizar la comunicación e interacción entre los miembros de las instituciones educativas.

La brecha de comunicación que existe entre las personas involucradas en las instituciones educativas es un problema que se repite constantemente en las instituciones educativas de todos los niveles del país debido a muchos factores (Arias, 2015). Actualmente, los directivos utilizan medios de comunicación como papeletas, cuadernos de control y comunicados, con los que las instituciones educativas tratan de abordar este tema, pero lo hacen de una manera tan convencional que no se observan resultados favorables. Por ello, este trabajo desarrolla una aplicación móvil para optimizar la comunicación e interacción entre los miembros de las instituciones educativas, con la que se comprueba el uso de la tecnología como una herramienta de trabajo que permite contribuir a mejorar la interacción en el nivel educativo.

El constante crecimiento del uso de los dispositivos móviles en las dos últimas décadas nos hace 
preguntarnos cómo otorgar valor agregado a esta herramienta que casi todas las personas utilizan para comunicarse y mantenerse conectados con el mundo entero. Las personas siempre llevan estos dispositivos a todos lados y están atentas a las notificaciones que reciben de diferentes aplicativos, ¿qué sucedería si alguna de estas notificaciones fuese una tarea que su hijo tiene que presentar en dos días o si se tratara de una reunión de urgencia, una calificación o una notificación de la inasistencia del menor a clases? El padre o tutor no tendría que esperar hasta llegar a casa para saber qué le ocurrió a su hijo en el transcurso del día. El padre o tutor debe estar informado de todas las actividades de su hijo en la institución educativa, para realizar un seguimiento personalizado al menor. Gracias a las bondades de la tecnología, se puede diseñar una herramienta que acerque a los padres y/o apoderados a la institución para lograr una armonía en las actividades. Las limitaciones identificadas en este estudio fueron la dificultad para encontrar locales donde implementar el modelo y la automatización de procesos para estandarizar el comportamiento de las instituciones educativas.

\section{Modelo de integración educomunicativa de apps móviles para la enseñanza y aprendizaje}

Las actividades educativas se dividen en 2 procesos: aprendizaje y enseñanza. Con las TIC, que en los últimos años han ido en aumento, y la cultura de innovación, se pretende crear nuevas metodologías que beneficien a las personas involucradas en las actividades educativas. Villalonga y Marta-Lazo (2015) muestran cómo deberían ser las actividades de enseñanza y aprendizaje; sostienen que es necesario adoptar un sistema de diálogos basados en la horizontalidad y bidireccionalidad en donde la palabra comunicación es clave, es decir, la comunicación entre los miembros involucrados en estas actividades debe ser clara y consistente para que todos se beneficien. Además, indican que la participación activa de los miembros, así como la colaboración e interactividad hará que las actividades de enseñanza y aprendizaje se lleven a cabo de manera eficiente.

A partir del modelo de aprendizaje de Villalonga y Marta-Lazo (2015) se intenta dar paso a la tecnología con el uso de dispositivos móviles accesibles a usuarios de todo nivel. Para tener una mejor idea del planteamiento del modelo de aprendizaje a través de un sistema móvil, se propone conocer a fondo las funcionalidades pedagógicas de las instituciones educativas; este modelo de aprendizaje se basa en el conocimiento impartido no solo por docentes, sino también por cualquier otra persona que brinde alguna información de un tema específico. El conocimiento está en construcción y, al estar interconectado entre los miembros, permite que la red se construya por sí misma y se vuelva cada vez más compleja. Cuando el estudiante investiga cómo resolver un problema planteado, accede a contenidos que este modelo registra y muestra de forma clara e incluso señala las herramientas que pueden utilizarse para concretar el conocimiento. Una vez que se accede a la información, esta se conecta de forma colaborativa con el de los compañeros y docentes, de este modo, la red de enseñanza crece de manera eficiente, lo que permite que el conocimiento esté en movimiento e interactúe con los usuarios. Una vez mostrado lo que el modelo de aprendizaje abarca, debe ser llevado a una plataforma móvil que brinda conectividad, este término tan simple que permite que el modelo sea más eficiente, puesto que las aplicaciones móviles estarán conectadas y los miembros de cada asignatura podrán tener un aprendizaje y enseñanza de acuerdo a la cultura de innovación que ofrece la tecnología (Villalonga y Marta-Lazo, 2015).

\section{Dispositivos móviles en educación superior y entornos personalizados de aprendizaje}

Para entender hacia dónde se quiere llegar, se presenta el término de m-learning que se apoya en e-learning y muestra una manera de mejorar los métodos de aprendizaje apostando por el aprendizaje y enseñanza móvil, puesto que la conectividad permite crear redes en donde los miembros de las entidades educativas interactúen. De aquí se realza la importancia de los smartphones, ya que cuentan con muchas herramientas (hardware y software) que se pueden explotar a favor de los objetivos educativos. Por medio de encuestas se obtendrán datos estadísticos fehacientes para conocer las bondades y particularidades de los distintos dispositivos móviles como la marca, la capacidad de memoria, costos de red, aplicaciones de preferencia, frecuencia del uso del móvil y demás, lo que permitirá observar y modelar la realidad a la que se le intenta dar un valor agregado. Estos datos son muy útiles para cualquier tipo de investigación referente a educación moderna, puesto que permite observar cómo se van formando las bases para afrontar una nueva etapa en la educación de una manera más tecnológica y sistematizada (Cataldi et al., 2012).

Estos datos estadísticos también muestran si los docentes están en condiciones de utilizar un smartphone, ya que no todos tienen las mismas necesidades al adquirir un dispositivo móvil. Incluso las necesidades formativas de los docentes son puntos que se deben tratar, puesto que 
el uso y/o método de enseñanza entre colegas varía. Tras los resultados de las encuestas, se reafirma lo que se pensaba acerca de la relación docente-tecnología: los alumnos tienen mayor ventaja en el tema de la tecnología en comparación con los docentes. Con este resultado, se tiene una visión más amplia de las necesidades de los usuarios y se plantean soluciones mediante situaciones de aprendizaje que colaboren con las actividades y que sean de provecho para las partes interesadas; así, se revela un nuevo paradigma denominado "aprender, desaprender $y$ reaprender", en el cual están integrados tantos los docentes como los alumnos.

\section{Aplicación móvil para el control escolar}

Las personas que tienen un celular inteligente olvidan que lo que tienen en las manos es una computadora portátil presta a ser utilizada como un solucionador de problemas de múltiples características. En la Universidad Politécnica de Francisco I Madero (UPFIM) de México, se detectaron dos puntos problemáticos para los alumnos, estos fueron: la atención del alumnado en sus deberes estudiantiles, desde el proceso de matrícula hasta los deberes propios de una asignatura (calificaciones, horarios e información general), y el tiempo de respuesta a las peticiones que hacían (Gomez, García y Reyna, 2016).

Para dar solución a estos problemas, en la UPFIM se propone desarrollar un sistema móvil que sirva para abordar tanto los problemas de la universidad como los diferentes tipos de realidades que son cambiantes; para ello se propone un método de desarrollo denominado Proceso Unificado de Desarrollo (RUP, por sus siglas en inglés). Una vez elegido el método, se elige el nombre C.A.S.A para el sistema que pretende, en cierta manera, solucionar los problemas de los alumnos de la universidad mencionada. Cuando se intenta utilizar el mismo sistema en otras instituciones aparece una barrera, pues cada entidad funciona con sus propios procesos que, con frecuencia, difieren de los estándares. La personalización es muy importante en esta etapa, para que puedan realizarse cambios pequeños (como los colores, logos, imágenes, recursos) o cambios mucho más notables (como cambios de procesos, quitar o agregar módulos) que permitan adaptarse a la realidad de cada institución. Se llega a esta conclusión porque el sistema se implementa en un colegio en donde se observa esa barrera. Se nos informa que el sistema está siendo probado en dos instituciones educativas: la universidad donde se desarrolló el sistema (UPFIM) y el Tecnológico de Monterrey,
Campus Hidalgo. Se advierte que el sistema, implementado en un sistema móvil al ser una herramienta muy utilizada en esa universidad, soluciona los problemas específicos de manera eficiente.

Se nos comunica la intención de implementar el sistema también en un colegio a modo de prueba.

\section{Análisis de las aplicaciones para dispositivos móviles inteligentes en apoyo al fortalecimiento académico en la DES DACI}

El bajo rendimiento de los estudiantes es un problema que preocupa a docentes, padres de familia e instituciones educativas, y se piensa que podría deberse a malos hábitos de estudio, problemas familiares, falta de motivación, entre otros. Otro factor notable y actual relacionado con este déficit es el uso desmedido de los celulares inteligentes, ya que se observa que los estudiantes están más pendientes del celular que de la clase, y, en los pasillos, están inmersos en las redes sociales, juegos y películas. Todas estas actividades los distraen, por lo que no les dedican tiempo a las actividades académicas o simplemente se olvidan de ellas, lo que provoca desventajas para su aprendizaje. Sin embargo, ¿qué sucedería si, en lugar de ser la causa del problema, el uso de los teléfonos inteligentes se convirtiera en un plus para mejorar el rendimiento académico? Eso, acompañado del uso de las aplicaciones, sería favorable para el ámbito educativo (Herrera y Buenabad, 2013).

En la actualidad, los smartphones son los teléfonos más utilizados por la comunidad estudiantil, dado que la demanda tecnológica aumenta cada vez más. En la Dependencia Académica Ciencias de la Información (DES DACI) de la Universidad Autónoma del Carmen (UNACAR), ubicada en Ciudad del Carmen, Campeche, México, existe una cantidad considerable de alumnos con bajo rendimiento académico; es por ello que tras una investigación usando una metodología cuantitativa se determinó que la universidad debía apostar por herramientas tecnológicas para capturar la atención de los alumnos. Los resultados fueron alentadores luego de implementar en la universidad las tecnologías de Evernote, Dropbox y My Homework; gracias a ello la universidad dio un primer paso hacia la sistematización de sus aulas y procesos de enseñanza y, al mismo tiempo, se reafirmó la idea de contar con una plataforma móvil. Aplicar estas tecnologías en el sector educativo no solo brindaría uniformidad en los procesos de cada institución, sino que también potenciaría el desarrollo académico de cada estudiante de educación superior al permitir la captura de datos interesantes para resolver actividades 
como tareas. Es emocionante imaginar lo que pasaría si en lugar de papeles, los estudiantes utilizaran Ms Word en sus celulares o guardaran sus archivos en Dropbox y ya no en un folder.

\section{Los docentes ante la integración educativa del teléfono móvil en el aula}

El tema de las TIC en el sector educativo debe abordarse desde las perspectivas de los involucrados en cada actividad educativa; a esto se le suma la reacción de los docentes, quienes presentan la información de las materias y el plan académico anual, y cuyo rol es fundamental en la inclusión de estas tecnologías en las aulas. Brazuelo et al. (2017) condujeron un estudio en donde participaron docentes de educación secundaria obligatoria de Las Palmas (Islas Canarias, España), en el cual se analiza el uso de los teléfonos inteligentes, así como las actitudes de los docentes frente a la integración de estos en los procesos académicos. Al ser consultados sobre su opinión acerca de este enfoque, se observó un cierto grado de desconfianza para reconocer nuevos métodos que acerquen a los estudiantes y optimicen su labor de educadores. Esto se observa más en los docentes adultos mayores, quienes desconocen los beneficios que estas herramientas podrían brindarles a ellos y a la institución. El reto es convencerlos de que la herramienta es funcional y es un facilitador de procesos para crear una mejor experiencia en las aulas.

La edad de los docentes que participaron en el estudio fue entre 36 y 51 años, y los resultados fueron diversos. Por una parte, un grupo de docentes considera que la tecnología avanza a mil por hora; además, manifiestan que la experiencia de los alumnos con los dispositivos comparada con la falta de formación en esta área por parte de los docentes y su edad es un factor en contra. Por otro lado, otro grupo de docentes un tanto más jóvenes considera el uso de smartphones como una herramienta que facilita, agiliza y dinamiza las comunicaciones entre padres, alumnos y demás miembros en general. Con este diagnóstico, se pretende llevar a cabo una búsqueda de información que sirva, a su vez, como punto de partida para futuras investigaciones de mayor precisión y como propuestas de mejora, así se dará un paso hacia la sistematización de los procesos educativos y se beneficiará a la sociedad mediante el uso de las TIC para la enseñanza escolar en todos los niveles.

\section{¿Es posible integrar los dispositivos móviles en educación primaria como recurso educativo?}

La integración de herramientas y recursos tecnológicos en el aula es una realidad viable que facilita la comunicación entre padres, profesores y alumnos. En cuanto al uso de recursos móviles, es importante destacar que su implementación por sí sola no propicia un desarrollo significativo de habilidades cognitivas, puesto que, frente a la situación académica actual, se requiere de una buena planificación docente en donde la interrelación de actividades sean provechosas para el alumnado (Gértrudix y Barroso, 2016).

La investigación basada en una encuesta a 68 estudiantes del $5^{\circ}$ curso de educación primaria de un colegio público situado en la provincia de Toledo, España, (Gértrudix y Barroso, 2016) aborda temas cruciales como el número de niños con acceso a un dispositivo móvil, tiempo de uso y tipo de aplicaciones de su preferencia. Al observar los resultados, se concluyó que los niños de 10 y 11 años contaban con dispositivos móviles (smartphones y tabletas), lo cual es sorprendente para su corta edad; se determinó también que las aplicaciones más utilizadas eran las de juegos y las de mensajería, asimismo, llama la atención el tiempo de uso del celular. Con base en esos resultados, se planteó la creación de una aplicación para el aprendizaje de contenidos históricos en el contexto de un aula de $5^{\circ}$ de primaria. Los datos obtenidos indican que los estudiantes de primaria tienen sus propias necesidades y han cambiado los juguetes por la tecnología; son los padres quienes deben estar vigilantes frente al uso desmedido de los dispositivos móviles. En algunos casos, los estudiantes solo pueden utilizar sus teléfonos móviles los fines de semana. En el ámbito educativo, es labor de los docentes motivar a los estudiantes pequeños a utilizar la tecnología de la mejor forma, de tal modo que no les resulte aburrido ni le den un mal uso.

\section{METODOLOGÍA}

Como primer método se realiza la recolección y consulta de material relacionado al tema, que se encuentra en bases de datos indexadas de los últimos años, con la finalidad de conocer el estado actual de la investigación. A continuación, se realiza el análisis y la clasificación del material científico directamente relacionado con el uso de aplicaciones móviles en el sector de educación, de este modo fue posible seleccionar los artículos a los que se hace referencia en la presente investigación.

Una vez se tienen el material y estudios referentes a las aplicaciones móviles utilizadas en el sector educativo, es momento del análisis y descripción del modelo a utilizar para iniciar con el desarrollo de la aplicación. Con este análisis se pretende tener un plan 
a seguir para intentar optimizar la comunicación e interacción entre los miembros de las instituciones educativas mediante el uso de una aplicación móvil.

A partir del modelo, se desarrolla la aplicación en un plazo razonable con las herramientas elegidas, y se definen roles específicos (profesores, padres, alumnos y personal administrativo) y funciones específicas para cada rol (tareas, notas, mensajes, asistencia, agenda, noticias, etc.). Cuando se termina la etapa de desarrollo, se pasa a la implementación de prueba del aplicativo móvil en instituciones seleccionadas para observar su adaptabilidad a los diferentes escenarios en beneficio de la educación en las instituciones educativas.

\section{RESULTADOS}

Se puede decir que las actividades académicas en la educación secundaria y superior competen principalmente a alumnos y docentes, mientras que, en el nivel primario, compete a padres, docentes y alumnos; entonces, la interacción es un tanto limitada (Fuentes, 2015). Es necesario conocer a profundidad la situación actual y apuntar hacia la automatización.

Con el sistema propuesto se pretender definir claramente a los usuarios e incluir al personal administrativo, a los auxiliares de educación y a todas las personas que formen parte del ámbito escolar.

Este aplicativo móvil cumple con los datos recolectados de la investigación, que se resumen en conectividad, usabilidad, interfaces amigables, portabilidad, simplicidad y complejidad óptima.

La aplicación móvil es una herramienta para transformar las clases en las aulas y modificar la forma de interactuar de los miembros de cada institución, destacando la comunicación constante en cada minuto del día para optimizar los procesos académicos y beneficiar a todas las personas involucradas.

Algunos padres tienen ideas anticuadas y ven con recelo el que sus menores hijos utilicen dispositivos móviles, pues los consideran una distracción. El reto es convencer a los padres de que la aplicación móvil está en el punto medio entre usabilidad y confianza (Pinos et al., 2018).

Por otro lado, son los docentes quienes interactúan de una manera más activa con los demás miembros de sus instituciones educativas; ellos ya tienen una rutina marcada por su experiencia. El sistema propuesto reestructuraría los procesos académicos y, mediante la aplicación móvil, facilitaría el trabajo de los docentes; sin embargo, con base en los datos estadísticos, muchas veces son reacios al uso de las TIC al no ser contemporáneos con ellas. Se requiere instruirlos en el uso de dispositivos móviles porque son conscientes del enorme potencial que tienen si se usan de la manera correcta.

Además de todo lo mencionado, se debe tener en cuenta que el personal administrativo puede interactuar con los padres, profesores, alumnos, auxiliares y tutores por medio de una plataforma donde se puede mantener una comunicación bidireccional; esto fomentaría la identificación de los miembros con la institución a la que pertenecen, así como la confianza.

La propuesta es una aplicación móvil que permita mantener la comunicación e interacción entre sus usuarios; enviar noticias, eventos, tareas, asistencias, mensajes e incidencias; compartir imágenes de momentos vividos en el aula y/o colegio; y agendar cada entregable de manera ordenada para que nada quede al azar. Así, se intenta dar un valor agregado a todo lo que pasa en el aula con una aplicación que posibilita la conexión las 24 horas del día por medio de notificaciones.

\section{Conceptualizar el aporte}

La finalidad del aportees analizar la funcionalidad del sistema, mostrar la arquitectura, y mostrar cómo será la interacción de los usuarios finales con la aplicación móvil. Es importante conocer cómo se comparten los datos entre los diferentes usuarios, dado que la interacción será vía teléfono móvil y es donde se concentrará la mayor carga de trabajo. La interacción móvil que se propone intenta ir a la vanguardia en un mundo en donde los smartphones tienen un excesivo protagonismo casi en cada momento del día; si se les diera una utilidad escolar que mejorara el rendimiento, se lograría un avance significativo en el ambiente educativo y también en las actividades que influyen y dependen de ella.

Una revolución educativa no solo consiste en cambiar un sistema, sino también el modelo escolar desde su origen. El modelo escolar vigente es poco inclusivo con las tecnologías actuales; existen tecnologías que intentan sistematizar las agendas escolares, pero que no se pueden aplicar de la mejor manera por el desconocimiento de las personas que integran cada parte del modelo. El sistema que se propone desea cerrar la brecha tecnológica existente en el ámbito educativo, así como la brecha que los mismos profesores o 
padres han creado como resultado del desconocimiento de las tecnologías.

Este sistema es concebido como una herramienta para cambiar el modelo escolar actual; no obstante, el sistema no podrá realizar todo por sí solo, sino que será necesario que cada entidad sea consciente de una transformación digital para llevar a cabo este gran cambio.

\section{Usuarios a quienes va dirigido}

Después de conocer la finalidad del sistema propuesto, se debe reconocer hacia qué personas va dirigido y cuáles son sus intereses, puesto que las necesidades de un profesor son distintas a las de un alumno.

Se identificaron 4 tipos de usuarios: alumnos, padres, profesores, y personal administrativo; cada uno de ellos responde a una necesidad distinta, por lo que se trató de identificar las funciones más críticas. Se muestran las funciones que cada tipo de usuario realiza en favor de la interacción y fluidez de comunicación entre las personas de cada institución educativa; además, se indica cómo las realizan ahora y cómo las realizarán con la implementación del sistema en su centro de estudio.

\section{Modelo de la arquitectura}

Como se observa en la Figura 1, el modelo identifica unos componentes que son de vital importancia para el correcto funcionamiento de la aplicación; estos componentes permiten dar soporte a una arquitectura que se prevé irá creciendo con el tiempo, debido a que los datos y el flujo de trabajo se volverán cada vez más complejos por la cantidad de usuarios que interactúa con el sistema en tiempo real.

\section{Base de datos}

La base de datos es importante porque ahí se aloja toda la información que el sistema necesita para funcionar. El servidor que se propone utilizar estará en la nube para que las conexiones se hagan desde cualquier lugar y a cualquier hora. Una buena base de datos permitirá que el servidor se mantenga operativo todo el día y, además, que sea escalable, algo que es muy relevante para el sistema que se propone.

La base de datos estará implementada en MySQL, la cual contará con tablas debidamente normalizadas que sostendrán toda la lógica que se quiere implementar.

Por el momento, la información se alojará en el servidor Hostinger, donde se conectará y donde estará funcionando la base de datos; desde ahí se podrán conectar todas las personas gracias a las API.

\section{Cloud Messaging Firebase}

Esta parte de la arquitectura (ver Figura 2) se apoya en la tecnología que brinda Firebase. Uno de los

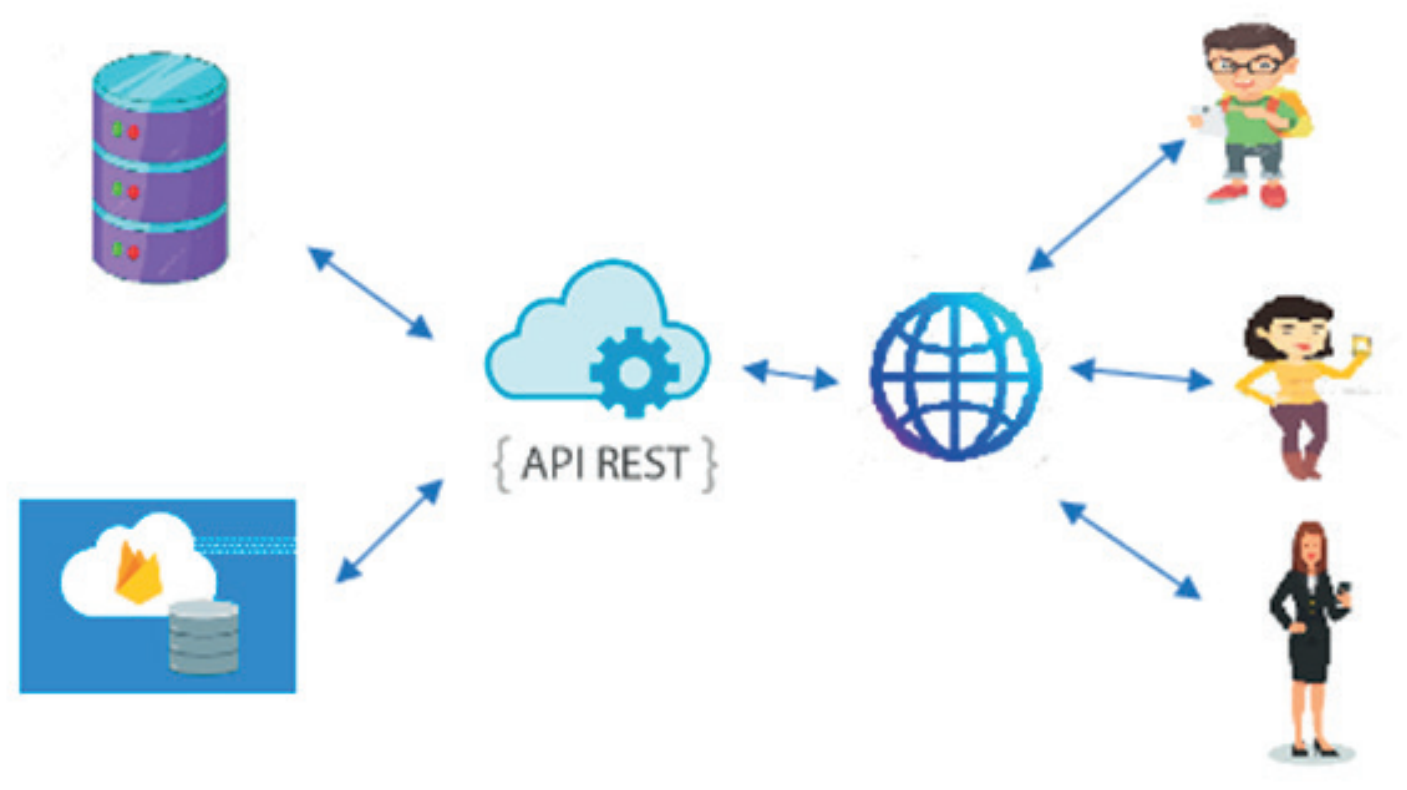

Figura 1. Modelo de arquitectura.

Fuente: Elaboración propia. 


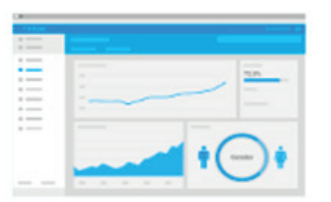

Notifications Console GUI

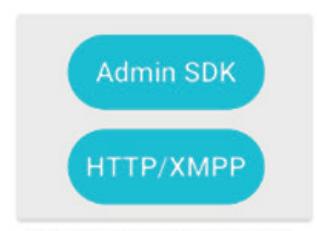

Trusted Environment
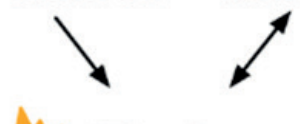

\section{Firebase}

Cloud Messaging

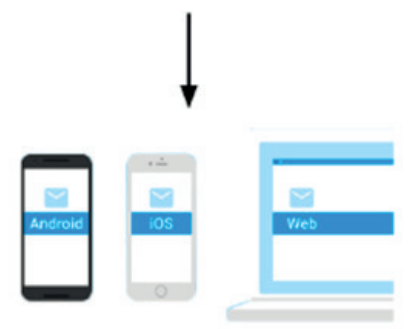

Figura 2. Cloud Messaging Firebase.

Fuente: Elaboración propia.

objetivos de este sistema es que los padres, alumnos y profesores estén siempre informados de las actividades, tareas o cualquier asunto que ocurra en el centro educativo, para evitar las excusas típicas como "No leí el cuaderno de control" o "Me olvidé de decirle a mi mamá". Para que el manejo de notificaciones se haga de una manera eficiente, es necesario apoyarse en Cloud Messaging Firebase.

Esta tecnología brinda las siguientes herramientas para el correcto funcionamiento del sistema:

\section{Token de registro}

Desde el móvil, se envía una solicitud de registro de token, el cual permite identificar al dispositivo móvil desde cualquier punto. Cada dispositivo móvil posee un token que es único en el mundo, esto permite mandar mensajes o notificaciones personalizadas mapeadas mediante un registro.

\section{Suscripciones por temas}

Otra herramienta es la suscripción por temas, que funciona de manera similar a lo que se observa cuando un usuario se suscribe a un canal de YouTube para recibir notificaciones. En el caso de esta aplicación de uso educativo, se trata de una herramienta muy importante para recibir las notificaciones de tareas, eventos, comunicados, chat grupal, o cualquier otro dato que haya sido ingresado. Esta parte es fundamental, puesto que es donde se apoya todo el concepto de notificar a cada usuario en tiempo real con tan solo mandar desde un servicio web un número ilimitado de notificaciones con el tema especificado.

Por ejemplo, cuando el profesor ingrese una tarea con el tema "TareasHorario5" todos los alumnos y padres que estén matriculados en el horario con identificador 5 serán notificados; de este modo todos estarán informados.

\section{FirebaseMessagingService:}

Este servicio, que estará funcionando en segundo plano en los dispositivos móviles, responderá a cada notificación y permitirá al usuario poner touch a cada notificación y lo redirigirá a una pantalla específica. El correcto funcionamiento de este servicio es crucial.

En resumen, Firebase posibilitará el control total de las notificaciones y, de este modo, hará que el proyecto sea escalable visto que estos servicios soportan una gran cantidad de datos.

\section{API REST}

Es necesario el desarrollo de las API para lograr una comunicación eficiente entre los dispositivos móviles y el servidor. Estas API están desarrolladas en PHP y java, lo que hace que su mantenimiento y actualizaciones sean procesos más sencillos, 
en esta parte es donde se da la lógica del modelo que se propone, interactuando directamente con la base de datos, trayendo datos cuando se solicitan mediante JSON, que son lo que devuelve para su tratamiento en el código Android.

Estas API también llaman a los servicios proporcionados por Firebase haciendo toda la lógica desde aquí. La conexión y la transformación a JSON. Un uso adecuado de esta parte de la arquitectura garantiza un correcto funcionamiento y mantenimiento eficiente.

\section{Internet}

Como todo sistema actual debe tener cobertura a internet, la conexión es lo más importante para enviar notificaciones o publicar cualquier evento. Se intenta consumir la menor cantidad de megas posible, y enviar la menor cantidad de solicitudes al servidor mediante el uso de HTTP.

El uso eficiente de este recurso le proporciona una solución óptima al usuario.

\section{Usuarios finales}

Es en esta etapa de la arquitectura cuando la aplicación móvil toma importancia, los usuarios interactúan con la aplicación móvil para, a su vez, interactuar con otros usuarios involucrados con cada institución educativa. Los profesores tendrán a su cargo cursos y alumnos quienes a su vez estarán vinculados a los padres.

Los dispositivos móviles estarán conectados entre sí y será sencillo recolectar los datos que se encuentren dentro de cada módulo. La arquitectura está preparada para que el usuario pueda acceder de manera local a la información almacenada en la base de datos local, aun cuando no tenga datos. Esto solo muestra la información vista con anterioridad.

Esta información será de utilidad para los usuarios porque podrán acceder a sus tareas, notas, eventos e incluso mensajes sin necesidad de tener acceso a internet, de esta forma la arquitectura se vuelve más robusta.

\section{Encuestas}

Para implementar este sistema, se consideró que la mejor opción era recolectar información entorno al mercado objetivo, es decir, los colegios de nivel primaria y secundaria. Para ello, se utilizaron encuestas, puesto que permiten obtener datos fehacientes de que lo que se quiere lograr tiene una base sustentada (Sánchez et al., 2014). Las encuestas fueron realizadas en el Cono Norte en tres etapas: la primera se realizó antes de lanzar el producto; la segunda, días después de lanzarse el producto; y la tercera, después de acabar el primer periodo de clases.

\section{- Antes de lanzarse el producto}

Esta parte de la encuesta fue elaborada para conocer cómo los alumnos, padres, profesores, directores y todas las personas incluidas en las instituciones educativas recibirán una aplicación móvil que intenta mejorar la interacción que existe entre ellos.

En el nivel de inicial, se observó que el módulo de alumnos no era tan necesario, puesto que solo 3 alumnos (de 5 años) contaban con algún tipo de smartphone, mientras que la totalidad de profesores sí cuenta con uno. La encuesta muestra también que tanto los padres como los profesores de este nivel se encuentran a la vanguardia de la tecnología. En el nivel de primaria, se observó que un pequeño porcentaje de profesores no contaban con un Smartphone, mientras que un gran número de alumnos ya contaban con uno. En este nivel, los estudiantes tenían un uso restringido de datos y, los que no tenían datos, afirmaban que en su casa accedían a internet. En el nivel de secundaria, se advirtió que los profesores, por tener más edad, a menudo contaban con celulares básicos en lugar de un smartphone, mientras que casi la totalidad de alumnos contaban con uno; además, con frecuencia, los profesores no tenían acceso a datos, pero se mostraron dispuestos a adquirir un plan.

También se observó que el poco conocimiento en el uso de alguna plataforma educativa supone una barrera para la transformación hacia la era digital. Son sobre todo los padres y los alumnos quienes tienen poco o nulo conocimiento del uso de dichas plataformas, mientras que los profesores o personal administrativo sí cuentan con experiencia al respecto por el uso de las aulas virtuales durante su preparación o que han visto por internet. Para los alumnos que no tienen acceso a un celular, la mejor forma de comunicación es mediante Facebook; mientras que, para quienes sí cuentan con un celular, la comunicación mediante WhatsApp es mejor.

Algo que causó sorpresa fue el interés que las personas mostraron ante a la oportunidad 
de tener un sistema que notifique algo tan simple como tareas (un pequeño modulo del sistema); este resultado hace suponer que lo ven como una posible solución a una necesidad. Los usuarios no desean contar con un nuevo correo y contraseña, comentaron que sería mejor tener un sistema parecido a WhatsApp en donde solo sea necesario registrarse una vez y que, al ingresar al sistema directamente pueda estar logueado, lo que facilita la rutina.

Los resultados obtenidos en las primeras encuestas son alentadores. De acuerdo con lo planteado, se desea entregar un sistema que cubra las necesidades básicas de los padres, alumnos y profesores para contribuir con el desarrollo académico; al mismo tiempo, se debe reconocer que esto no solo es una aplicación, sino un cambio en el modelo de aprendizaje y comunicaciones.

\section{- Días después de lanzarse el producto}

Los resultados mostraron que el sistema tuvo acogida entre los usuarios y que las personas encuestadas fueron debidamente informadas por los colegios sobre el uso de la aplicación en favor de la transformación digital. Se observó una aceptación superior al $90 \%$ en todos los tipos de usuario identificados, gracias a esto se puede saber que se va por un buen camino. Asimismo, se observó un 92\% de aceptación por parte de los profesores, algo que sorprendió en ese momento, pero que sería de utilidad para identificar problemas o flujos que no fueran evidentes. Los alumnos, padres y personal administrativo se mostraron conformes, puesto que su función cumple a cabalidad los parámetros que se proyectaban. Se obtuvo un $100 \%$ de aprobación en cuanto a facilidad de uso y sencillez en las pantallas.

- Se debía probar si el sistema estaba siendo capaz de notificar en tiempo real cada vez que había una interacción; esto era muy importante ya que dependíamos del servicio de Firebase. Fue muy grato saber que los usuarios estaban siendo notificados correctamente, lo que comprobó la usabilidad y funcionalidad del sistema.

- Después de cerrar el primer periodo de clases (26 de agosto el 2018)

Esta tercera etapa de la encuesta fue dirigida a los profesores, ya que, después de cerrar el primer periodo (bimestre o trimestre), era necesario saber si el uso del sistema había sido el esperado y la reacción de los usuarios, satisfactoria. Con esos fines, se elaboró una encuesta de 5 preguntas dirigida a 50 profesores de los diferentes colegios con los que se trabajó. Los resultados obtenidos mostraron que los profesores en su totalidad coincidieron en que el módulo de tareas fue de mucha utilidad. Incluso opinaron que el sistema facilitaba su trabajo, puesto que podían mandar las tareas a todos sus alumnos y padres en cuestión de segundos; de esta forma, se logró reducir el tiempo de envío y mantener la comunicación.

También se observó que el módulo de notas fue muy útil para los profesores porque cumplía con la función de comunicar. No es un súper módulo de cálculo de promedios, sino un registro de notas; los profesores lo entendieron y fue de utilidad, pero queda la promesa de optimizar el sistema y asimilarlo a un aula virtual.

Los profesores quedaron contentos con lo presentado, y aunque el sistema tiene potencial aún existen aspectos por mejorar. Esta es la primera versión del sistema y cumple funcionalmente, deja contentos a los usuarios y, por tanto, se coloca la primera piedra para la transformación digital.

Las encuestas presentadas fueron tomadas en tres etapas diferentes, lo cual permite tener una gran visión de lo que el sistema es capaz de brindar en las instituciones educativas. Gracias a esos datos se puede observar la transformación digital.

\section{Desarrollo de la aplicación}

\section{Interfaces}

Para dar una mejor visualización al sistema planteado, se presentan las interfaces diseñadas para el sistema de interacción y comunicación escolar al cual hemos denominado MottyApp.

En las Figuras 3 y 4, se muestra la pantalla que el usuario observará cuando descargue la aplicación de la Play Store. En esta pantalla se ingresa el código de usuario que se le proporcionará cuando adquiera el sistema, una vez ingresado el código, el sistema buscará asociar al usuario con el código y mostrará la Figura 5.

Las figuras 6 y 7 representan las pantallas principales de acuerdo con el usuario al cual fue asignado. Cada usuario tiene sus propios módulos, algunos 


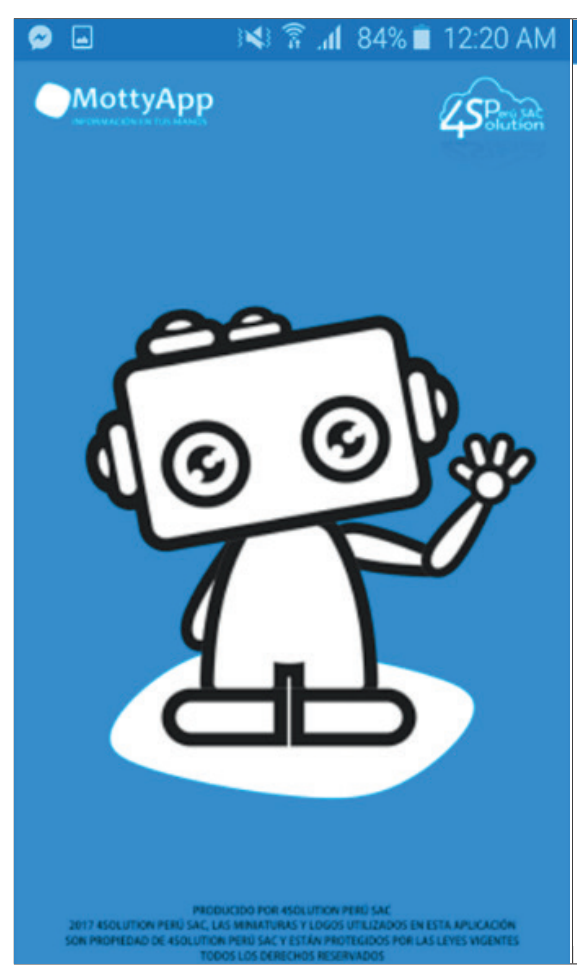

Figura 3. Splash de inicio de sistema.

Fuente: Elaboración propia.

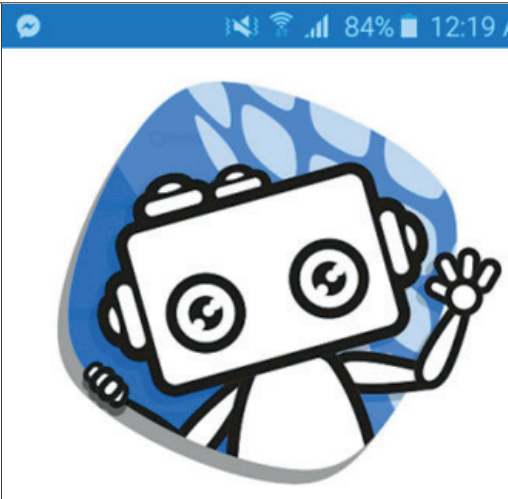

|lngrese código de usuario

Nombre del usuario:

Nombre del Colegio:

Cumple el rol de

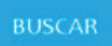

Figura 4. Pantalla de registro. Fuente: Elaboración propia.

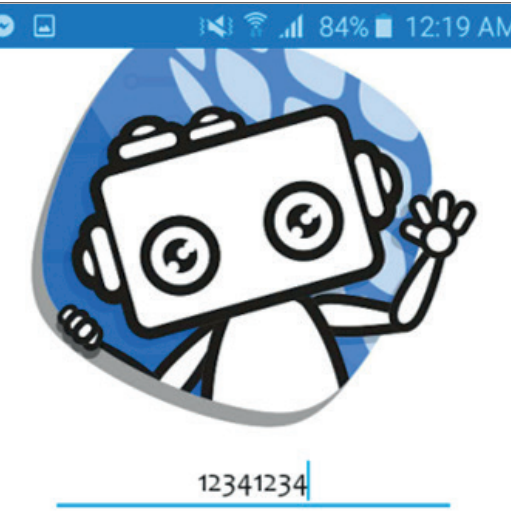

Nombre del usuario: Torres Davila George Deave

Nombre del Colegio: Jean Paul Sartre

Cumple el rol de profesor

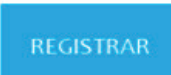

Figura 5. Pantalla de confirmación de registro.

Fuente: Elaboración propia.

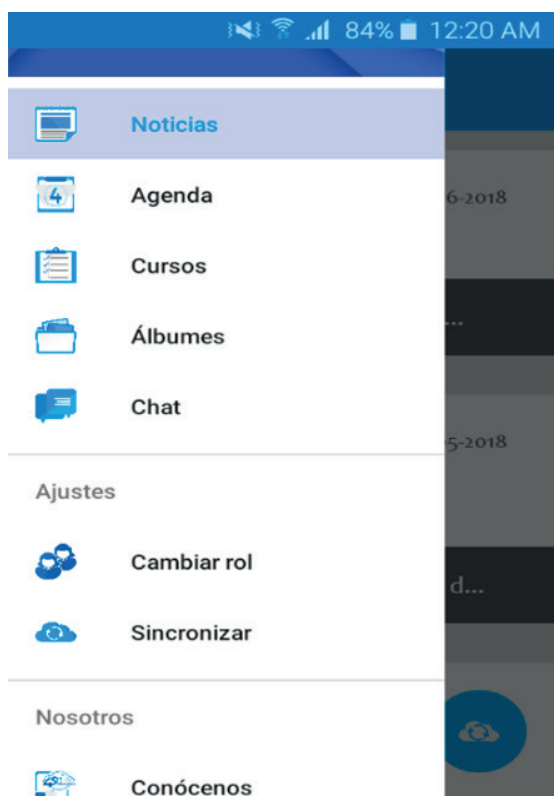

Figura 6. Pantalla principal (alumno). Fuente: Elaboración propia.

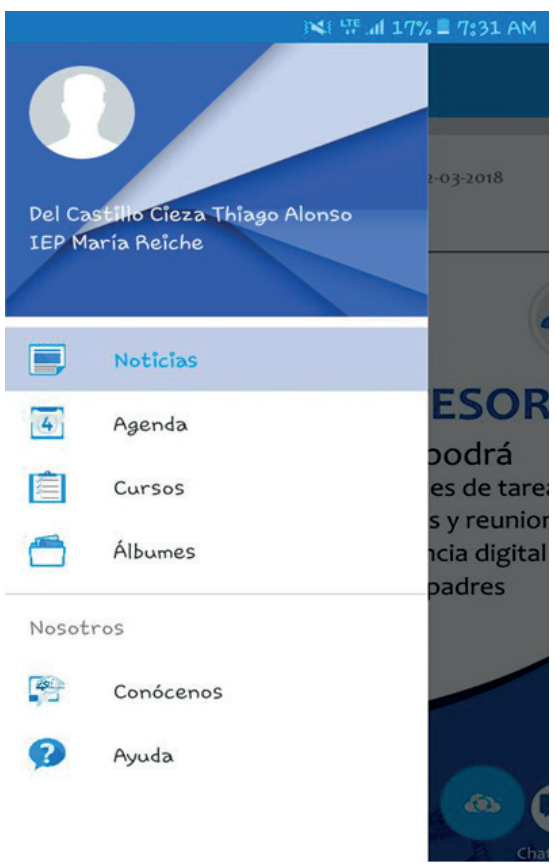

Figura 7. Pantalla principal (profesor).

Fuente: Elaboración propia. 
distintos en su funcionalidad y otros, similares. La barra lateral se diseñó para la comodidad del usuario, ya que es mucho más sencillo tener todas las opciones en la pantalla principal que evita el flujo innecesario del sistema.

La Figura 8 representa la pantalla de noticias, la cual será deslizable hacia arriba, simulando Facebook, para que el usuario se sienta cómodo y más familiarizado con la aplicación.

La Figura 9 representa el módulo de álbum que mostrará los álbumes creados por los usuarios de una manera organizada con la etiqueta "nuevo", para que la red se mantenga enterada del número de fotos y cantidad de álbumes existentes. Cada álbum estará asociado a un curso o sección, al cual solo tendrán acceso las personas autorizadas; no existe un límite para agregar fotos en los álbumes.

La Figura 10 representa la pantalla de agenda, una de las más importantes del sistema, ya que recolectará toda la información para mostrarla en un calendario en donde se utilizarán distintos colores con sus propios significados para señalar si se trata de una tarea, un evento, reunión, etc.

Para ver qué hay dentro de cada día solo se es necesario un touch para observar los pendientes de ese día organizados en una sola pantalla.
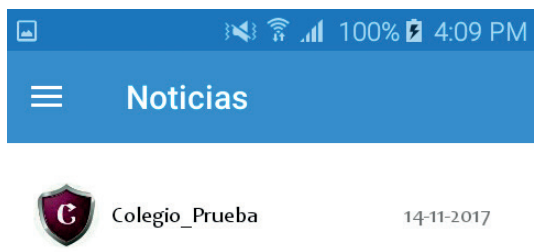

Reconocimiento de mejores proyectos

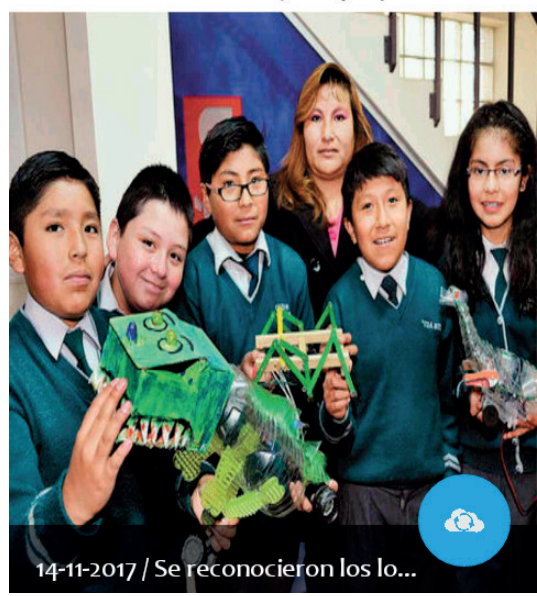

Figura 8. Pantalla de noticias Fuente: Elaboración propia.
La Figura 11 representa la pantalla de cursos que tendrá la lista de cursos ordenados alfabéticamente y asociados a una imagen para facilitar la navegación para el usuario.

Las imágenes que se ponen son dinámicas y cambian de acuerdo con la estación del año. Estos cursos son los que el alumno lleva; si eres padre, son los que tu hijo lleva; y, si eres profesor, son los cursos en los que dictas clases. Entonces, las imágenes permiten ordenar y sistematizar la información.

Las Figuras 12 y 13 representa los menús dentro de los cursos y tienen todas las funcionalidades bien definidas. Es un menú muy interactivo y lleno de colores para ser atractivo a las personas; cada curso tendrá su propio menú de tareas, incidencias, eventos, asistencias para así mantener un orden. Se plantea este menú deslizable hacia arriba para que el usuario se mantenga familiarizado, y que la transición a esta herramienta sea sencilla.

Las Figuras 14, 15, 16 presentan algunas de las pantallas que se verán dentro del menú de cursos, las cuales siguen la misma línea para así mantener la uniformidad, lo que representa en gran manera lo que se está proponiendo, un sistema de organizado, útil y que sea amigable.
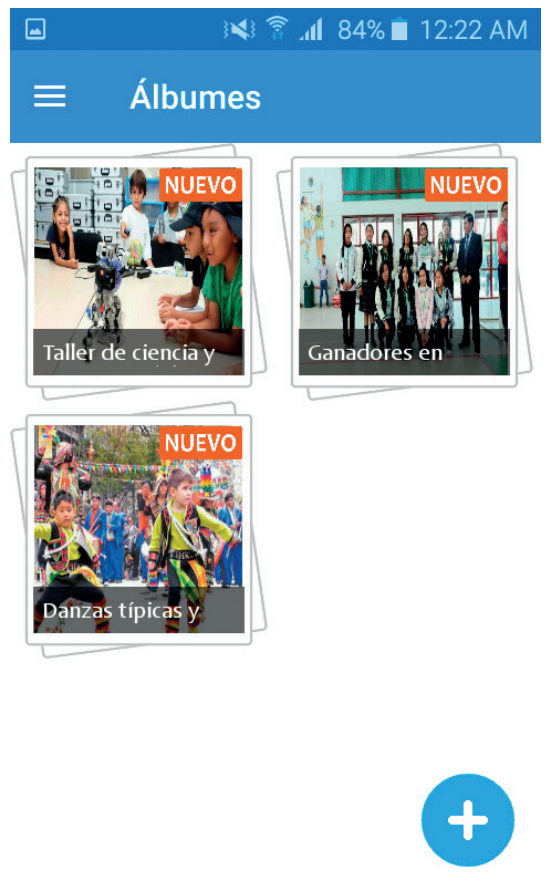

Figura 9. Pantalla de álbum Fuente: Elaboración propia. 


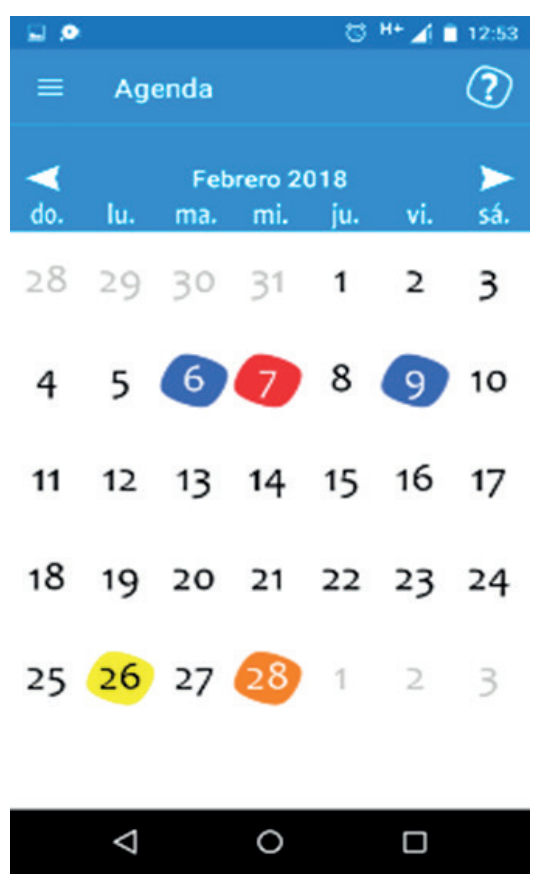

Figura 10. Pantalla de agenda. Fuente: Elaboración propia.

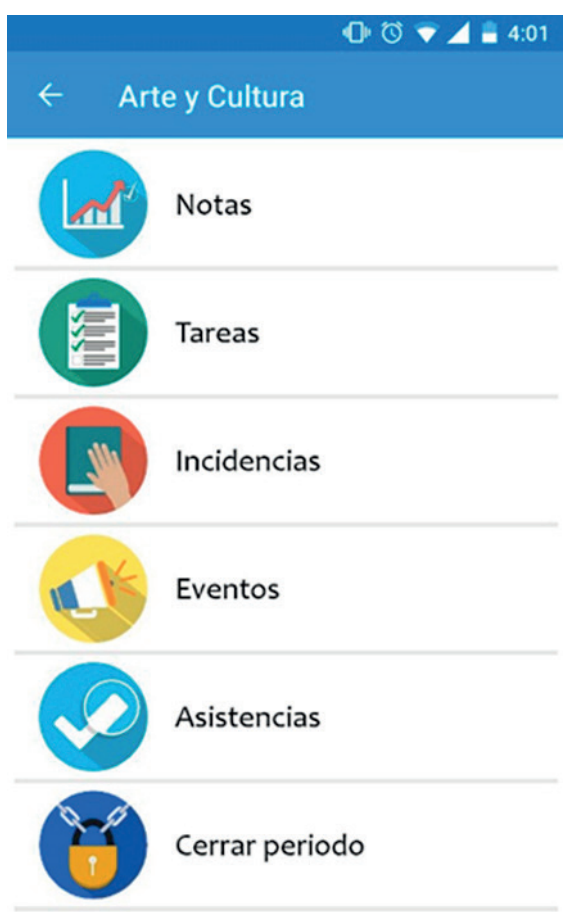

Figura 12. Menú de cursos (profesor). Fuente: Elaboración propia.

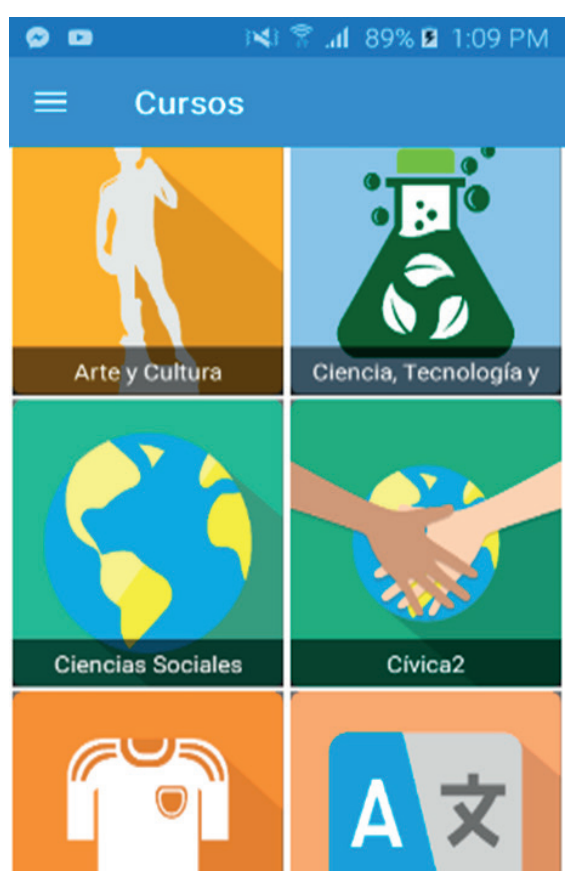

Figura 11. Pantalla de cursos. Fuente: Elaboración propia.
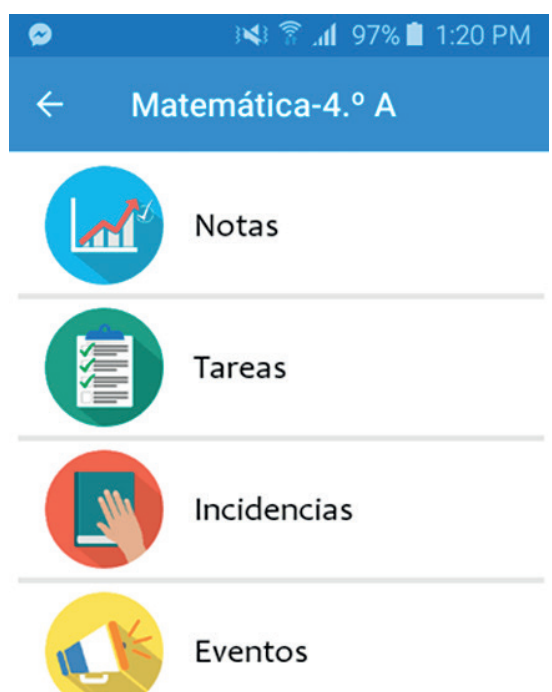

Asistencias

Figura 13. Menú de cursos (padre y alumno).

Fuente: Elaboración propia. 
Las Figuras 17 y 18 representan el módulo que los padres y profesores tendrán, así como el chat, el cual contribuirá con la solución del problema de comunicación e interacción al acortar la brecha que existe actualmente; su diseño es amigable y dinámico. La Figura 19 representa al módulo de GPS de los padres, el cual intenta acercarlos a los alumnos, pues el padre conocerá en tiempo real la ubicación exacta de su hijo con tan solo entrar al módulo, lo que le permitirá monitorear a su menor hijo.

\begin{tabular}{l}
\hline Asistencias \\
$\begin{array}{l}\text { Díaz Vásquez Ximena } \\
\text { Mariana }\end{array}$ \\
$\begin{array}{l}\text { Giraldo Duque Carlos } \\
\text { Augusto }\end{array}$ \\
\hline $\begin{array}{l}\text { González Tobón } \\
\text { Yolanda Sonia }\end{array}$ \\
\hline $\begin{array}{l}\text { González Zárate } \\
\text { Héctor Iván }\end{array}$
\end{tabular}

Figura 14. Asistencias.

Fuente: Elaboración propia.
Figura 15. Eventos.

Fuente: Elaboración propia.
Figura 16. Listado de pendientes. Fuente: Elaboración propia.

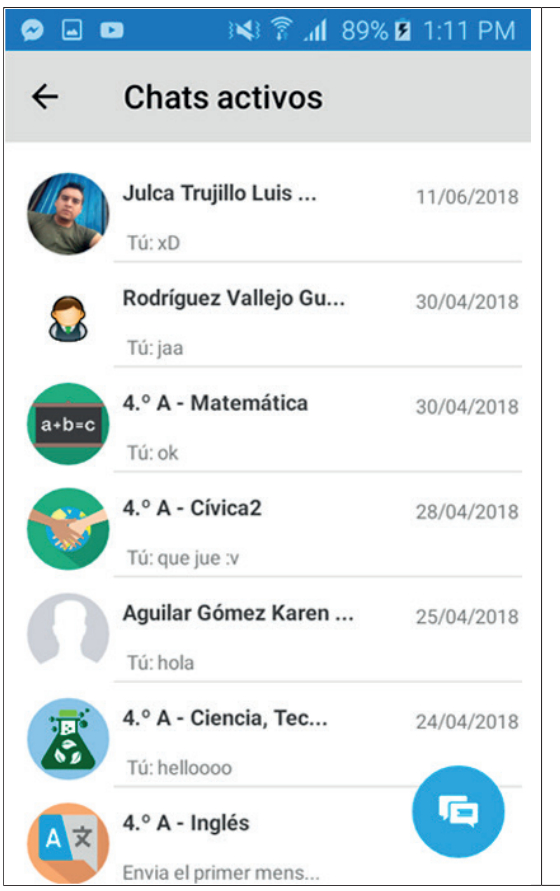

Figura 17. Lista de chat. Fuente: Elaboración propia.

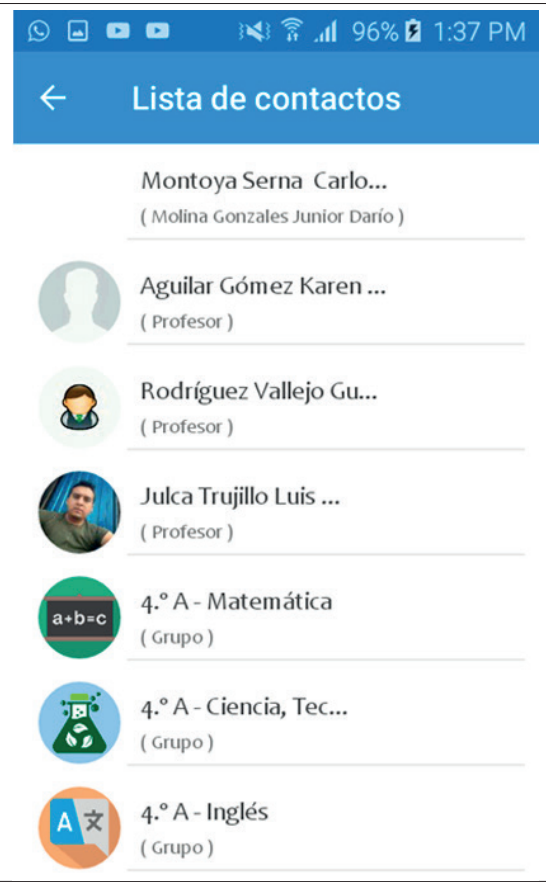

Figura 18. Lista de contactos. Fuente: Elaboración propia.

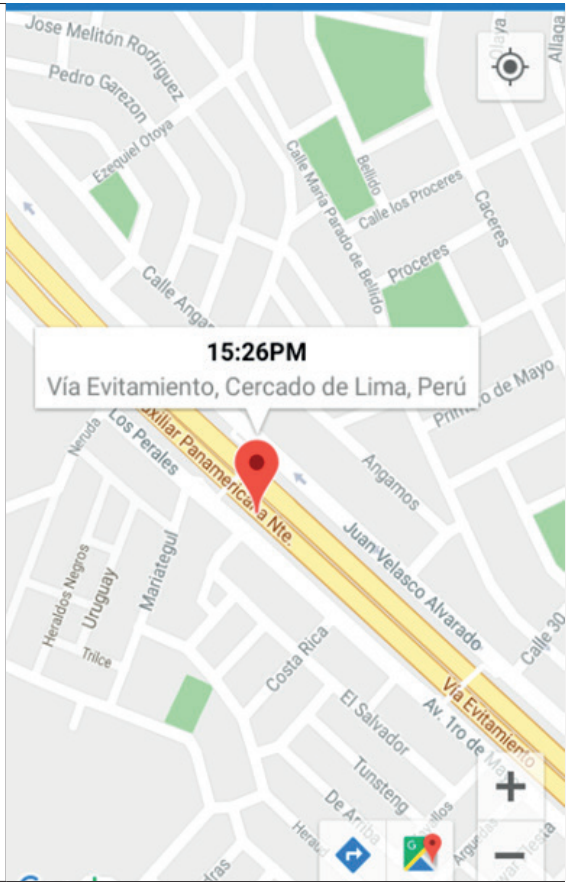

Figura 19. Ubicación del alumno Fuente: Elaboración propia. 


\section{DISCUSIÓN}

Al iniciar este proyecto de investigación, se advierte que algunas instituciones educativas utilizan aplicaciones de mensajería para cumplir con sus actividades con la intención de mantener la comunicación con los participantes de las aulas.

Usar las aplicaciones que existen en el mercado para realizar actividades propias de una institución educativa hace tediosa la navegación y recepción de pendientes, no se puede mantener un orden y no todas las personas están familiarizadas con ellas. El presente estudio busca mantener un orden para cada actividad, así se trate de un pendiente agendado, notas, tareas, comunicados, mensajería, etc., que permita tener todo en un solo lugar y que sea intuitivo y eficaz, es decir, de uso amigable para las personas de cualquier edad. En tal sentido, se pretende dar un paso más en el uso de las tecnologías en las instituciones educativas para así contribuir con el crecimiento académico de los estudiantes, así como con la comodidad de los profesores y de los padres.

\section{CONCLUSIONES}

Luego del estudio realizado, se tiene una visión más clara del sector educativo y se observa que existe un desfase en sus procesos de comunicación. Al ser este un mundo tecnológico, se debe aprovechar mucho más la tecnología que todos los días tenemos a la mano, es decir, los smartphones.

La comunicación e interacción entre las personas involucradas en una institución educativa es de por sí mala, sin embargo, con el sistema propuesto se da un gran paso hacia la mejora y se responde a una necesidad que, por lo general, no recibe la atención requerida. Gracias a la información proporcionada antes de la implementación del aplicativo móvil y a las personas encuestadas e informadas sobre los beneficios de este sistema, sumándole que han interactuado funcionalmente, se puede decir que el sistema aporta soluciones al tan desgastado tema de comunicación entre padre-alumno-profesor.

El aplicativo móvil tiene sus limitantes, pero es el punto de partida hacia la transformación digital. Los módulos fueron desarrollados pensando en las necesidades de las personas involucradas. La propuesta es una manera sencilla de abordar dichas necesidades y contempla la posibilidad de mejorar los módulos, así como de reconocer nuevas funcionalidades que surjan posteriormente. El sistema cumple con lo que se estipula en el problema y el estudio se realizó de manera formal.

\section{REFERENCIAS BIBLIOGRÁFICAS}

[1] Arias, W. (2015). Tecnologías de la información y la comunicación en colegios públicos y privados de Arequipa. Interacciones, 1(1), 1128.

[2] Brazuelo, F., Gallego, D., y Cacheiro, M. (2017). Los docentes ante la integración educativa del teléfono móvil en el aula. RED. Revista de Educación a Distancia, 52(6). Recuperado de http://dx.doi.org/10.6018/red/52/6

[3] Cataldi, Z., Méndez, P., Dominighini, C., y Lage, F. (2012). Dispositivos móviles en educación superior y entornos personalizados de aprendizaje. XIV Workshop de investigadores en ciencias de la computación, 1014-1018.

[4] Díaz, J., Ucán, J., Aguileta, A., y Toscano, A. (2016). Asistente escolar para los estudiantes de Ingeniería de Software: una aplicación móvil. ReCIBE, 5(3).

[5] Fuentes, O. (2015). La organización escolar. Fundamentos e importancia para la dirección en la educación. VARONA, 61, 1-12.

[6] Gértrudix, F., y Barroso, M. (2016).¿Es posible integrar los dispositivos móviles en educación primaria como recurso educativo? En J. Gómez, E. López, y A. Martín (Eds.), Advances and Innovations in Educational Research (págs. 35-43). San Juan, Puerto Rico: UMET Press and editors.

[7] Gomez, M., García, E., y Reyna, O. (2016). Aplicación Móvil para Control Escolar. Revista de Aplicación Científica y Técnica, 2(4), 1-5.

[8] Herrera, B., y Buenabad, M. (2013). Análisis de las aplicaciones para dispositivos móviles inteligentes en apoyo al fortalecimiento académico en la DES Ciencias de la Información. Revista Iberoamericana Para La Investigación y El Desarrollo Educativo, 11.

[9] Mendoza, M. I. (2014). El teléfono celular como mediador en el proceso de enseñanzaaprendizaje. Omnia, 20(3), 9-22.

[10] Molina, A., Roque, L., Garcés, B., Rojas, Y., Dulzaides, M. y Ganén, M. (2015). El proceso de comunicación mediado por las tecnologías de la información. Ventajas y desventajas en diferentes esferas de la vida social. Medisur, 13(4).

[11] Muñoz, E., Fernández, D., Gómez, J., y Arevalo, C. (13-15 de agosto de 2015). Impacto de Aplicaciones para Dispositivos 
Móviles: Un estudio de Aceptación [artículo científico]. Twenty-First Americas Conference on Information Systems, San Juan, Puerto Rico.

[12] Paredes-Parada, W. (2019). Brecha en el uso de tecnologías de la información y comunicación (TIC) básicas y modernas entre estudiantes y docentes en universidades ecuatorianas. Revista Educación, 43(1). Recuperado de https://doi.org/10.15517/revedu.v43i1.27423

[13] Pinos, N., Hurtado, S., y Rebolledo, D. (2018). Uso del teléfono celular como distractor del proceso enseñanza - aprendizaje. Enfermería Investiga, Investigación, Vinculación, Docencia y Gestión, 3(4).
[14] Sánchez, B., Diez, G., y Buenabad, M. (2014). El uso de los teléfonos móviles, las aplicaciones y su rendimiento académico en los alumnos de la DES DACI. Revista Iberoamericana Para La Investigación y El Desarrollo Educativo, 12.

[15] Villalonga C., y Marta-Lazo, C. (2015). Modelo de integración educomunicativa de 'apps' móviles para la enseñanza y aprendizaje. Pixel-Bit Revista de Medios y Educación. (46), 137-153. 


\title{
Design and Development of an Educational Mobile Application to Optimize Communication and Interaction Between Members of Educational Institutions in Real Time
}

\author{
MARÍA Elena RuIZ RIVERA ${ }^{1}$ \\ GeORGe TORRES DÁvila ${ }^{2}$ \\ EDGAR RuIz LIZAMA ${ }^{3}$
}

Received: 15/01/2021 Aceptado: 26/05/2021 Published: 26/07/2021

\begin{abstract}
The objective of this research is to optimize the communication and interaction between members of educational institutions, using a mobile application to improve service quality of the institution. By using the database with the information collected by the institution, as an important source for the integration and collection of education sector information, each institution is considered as an entity; it could be said that all the people who interact with it are the population, which needs to be connected and communicated with each other to subsist. Based on the exposed premises, a mobile application is designed to gather all users that interact with the same institution in a single platform. Thus, users are connected and communicated with each other by determining specific roles, which will have specific functions enabled for each type of user
\end{abstract}

Keywords: mobile application; mobile system; Cloud; Cloud Messaging Firebase.

\section{INTRODUCTION}

The idea of developing an application arose from the intention of improving communication and interaction between the members of an educational institution to bring them closer together so that they can interact more effectively for their own benefit and for that of their institution.

Part of the motivation for conducting this study was to enhance students' academic performance by improving basic but fundamental processes for educational institutions, such as communication and interaction between its members. Should those involved in the academic development of each student be brought closer to such an extent that they are able to advance towards academic goals, academic development would be more likely to be as expected and optimal for the student's education, as interaction would be personalized and increasingly closer.

In order to propose a possible solution to this problem, kindergarten, elementary and secondary school students, parent and teachers were surveyed. The responses obtained revealed the aspects to be improved, ranging from homework assignments, to personalized question answering, to attendance and performance report cards, which should be accessible at any time; it was also revealed that parents are not usually notified when an assignment or a grade has been given, mainly because students do not inform their parents, creating a gap between students, parents and teachers. For all these reasons, we propose a mobile application that adapts to the needs of the educational institution, minimizes the gap and, at the same time, optimizes communication and interaction. Another important aspect is that

1 Degree in Computer Science. Currently working as professor at the School of Systems Engineering and Computer Science, Universidad Nacional Mayor de San Marcos. (Lima, Peru). ORCID: https://orcid.org/0000-0003-3300-7068

Corresponding author: mruizr@unmsm.edu.pe

2 Graduated from the School of Systems Engineering and Computer Science of Universidad Nacional Mayor de San Marcos. Business Intelligence Analyst. (Lima, Peru). ORCID: https://orcid.org/0000-0003-0086-5066

E-mail: george.deave.95@gmail.com

3 Master in Computer Science from Pontificia Universidad Católica del Perú. Currently working as professor at the School of Industrial Engineering, Universidad Nacional Mayor de San Marcos. (Lima, Peru).

ORCID: https://orcid.org/0000-0001-9403-1358

E-mail: eruizl@unmsm.edu.pe 
it will also be aimed at parents and teachers, most of whom are not familiar with new technologies (Muñoz et al., 2015), which is why we propose a user-friendly and easy-to-use mobile application. The proposal attempts to bridge the gap between people from the same institution, through the intuitive and easy use of a mobile application whose main pillars are communication and interaction (Paredes-Parada, 2019).

This mobile application must be different from the tools used today, such as messaging applications, in which assignments and/or tasks are lost among countless messages or where everything is placed in a single chat room (Herrera \& Buenabad, 2013). The necessary tools must be provided for education to be the optimum, as well as assistance in the most basic activities to improve student learning (Gértrudix \& Barroso, 2016).

Human beings are sociable by nature and need to know, investigate and be informed, so much so that communication has undergone several changes concerning methods, types and methodologies; large-scale studies show that both information and communication are vital to achieve significant changes in the life of human beings (Molina et al., 2015). Communication is no longer only done verbally, orally or in writing, because now technology provides human beings with connectivity and interconnectivity allowing them to visualize images and to hear sounds in real time, which is applicable on a global scale (Cataldi et al., 2012). In recent years, interconnectivity between people around the world has increased in almost all sectors of the population and at all socio-cultural levels, thanks to the use of technology and the proper management of tools such as mobile devices called "smartphones", which every day are acquired by millions of people and used mostly for communication and information worldwide thanks to the internet (Gomez et al, 2016).

The aim of this research project is to optimize communication and interaction between members of educational institutions, i.e., to make the teacher-parent, principal-teacher relationship more open and meaningful, using mobile devices. Educational institutions integration achieved using mobile devices for teaching and learning is gaining momentum. Villalonga and Marta-Lazo, 2015 mention that in order to successfully carry out each stage of their learning model, certain already consolidated tools such as Google Drive, Facebook, Twitter, etc. must be used; the authors state that these tools must be used separately for each module of their learning model, this must now change since these functionalities can be included in a single app and fulfill the functions that they perform separately. As real statistical data were available after conducting surveys and having ordered data, two roles were defined: that of teachers and that of students. Both groups were interviewed to learn about the reality of educational activities, and the results obtained were encouraging; questions were asked about their type of mobile device, the company contracted, the most frequently used applications, the cost of the network, frequency of phone use, etc. (Mendoza, 2014). These data are very useful for any type of research concerning modern education, as they allow observing how the bases are being formed to face a new stage in education in a more technological and systematized way (Cataldi et al, 2012). The use of mobile devices as a tool that serves as a mediator between teaching and learning should be seen as an opportunity to add value to education (Mendoza, 2014). This paper focuses on the use of mobile devices to optimize communication and interaction among members of educational institutions.

The communication gap that exists between the people involved in educational institutions is a recurring problem in educational institutions at all levels in the country due to numerous factors (Arias, 2015). At present, managers use means of communication such as ballots, control notebooks and memos, with which educational institutions try to address this issue, yet it is done in such a conventional way that favorable results are not observed. Therefore, we develop a mobile application to optimize communication and interaction among the members of educational institutions, which demonstrates the use of technology as a working tool that can contribute to improve interaction at the educational level.

The steady growth of mobile devices over the last two decades drives us to look for ways to add value to this tool that almost everyone uses to communicate and remain connected to the world. People always carry these devices everywhere and are attentive to all notifications they receive from different applications. What would happen if one of these notifications was an assignment that your child has to submit within two days or if it was an urgent meeting, a grade or a notification of the student's absence? Parents or guardians would not have to wait until they get home to find out what happened to their child during the course of the day. Parents or guardians should be informed of all their child's activities at school, so that they can follow up on their child's progress. Technological advantages make it possible to design a tool that brings parents and/or guardians closer to the institution in an attempt to reach harmony. The limitations identified in this study were difficulties in finding institutions to implement the model, 
and the automation of processes to standardize the behavior of educational institutions.

\section{Educational-Communicative integration model of mobile apps for teaching and learning}

Educational activities are divided into 2 processes: learning and teaching. New methodologies that benefit those involved in educational activities are intended to be created using ICTs, which have been on the rise in recent years, and the culture of innovation. Villalonga and Marta-Lazo (2015) show the way teaching and learning activities should be; they maintain that it is necessary to adopt a system of dialogues based on horizontality and bidirectionality where communication is key, i.e., communication between the members involved in these activities must be clear and consistent so that everyone benefits from it. They further point out that the active participation of the members, as well as cooperation and interactivity, will ensure that the teaching and learning activities are efficiently performed.

Based on the learning model of Villalonga and Marta-Lazo (2015), we intend to give way to technology with the use of mobile devices accessible to users of all educational levels. In order to better understand the approach of the learning model by means of a mobile system, an in-depth knowledge of the pedagogical functionalities of educational institutions is required; this learning model is based on the knowledge imparted not only by teachers, but also by any other person who provides information on a specific subject. Knowledge is under construction and, as it is interconnected among members, it enables the network to build itself and become increasingly complex. During the research on how to solve a given problem, the student accesses contents that this model records and clearly shows, and even shows the tools that can be used to make the knowledge concrete. Data, once accessed, is connected with that of peers and teachers, so that the learning network grows efficiently, allowing information to circulate and interact with the users. It then must be transferred to a mobile platform that provides connectivity, a simple term that allows the model to be more efficient, considering that the mobile applications will be connected and the participants of each subject will be able to learn and teach according to the culture of innovation offered by technology (Villalonga \& Marta-Lazo, 2015).

\section{Mobile devices in higher education and person- alized learning environments}

To understand the direction of the study, we introduce the term m-learning, which is based on e-learning and provides a way to improve learning methods opting for mobile learning and teaching, since connectivity allows the creation of networks where members of educational institutions can interact. From this, emphasis is placed on the importance of smartphones, for they are equipped with many tools (hardware and software) that can be exploited in favor of educational objectives. Reliable statistical data will be collected using surveys to learn about the benefits and particularities of various mobile devices such as brand, memory capacity, network costs, users' preferred applications, usage frequency and others, thereby making it possible to observe and model the reality to which we intend to give added value. These data are very useful for any type of research related to modern education, as they allow observing how the bases are being formed to face a new stage in education in a more technological and systematized way (Cataldi et al., 2012).

Data also provide further evidence of whether teachers are in a position to use a smartphone, as not everyone has the same requirements when acquiring a mobile device. Furthermore, teachers' training requirements are aspects that need to be considered, since the use and/or method of teaching among colleagues varies. The results of the surveys confirmed our previous thoughts about the teacher-technology relationship: students have a greater advantage when it comes to technology compared to teachers. A more comprehensive picture of the users' needs is obtained from the results, and solutions are proposed involving learning situations that complement the activities and are of benefit to the parties involved. A new paradigm called "learn, unlearn and relearn" is thus revealed, in which both teachers and students are integrated.

\section{Mobile application for school management}

People with smartphones forget that the device they have in their hands is a portable computer that can be used as a multi-featured problem solver. At Universidad Politécnica de Francisco I Madero (UPFIM) in Mexico, researchers detected two problem areas for students, these were: students' attention to schoolwork, from the enrollment process to the homework (grades, schedules and general information), and the response time to the requests they made (Gomez et al., 2016).

At UPFIM, the development of a mobile system is proposed to address both the university's problems and the different types of changing realities; to this end, a development method called the Rational Unified Process (RUP) is proposed. Upon choosing 
the method, the system that is intended to solve the problems of the students of the aforementioned university is named C.A.S.A. Attempts to use the same system in other institutions encounter barriers, as each entity has its own processes, which often differ from the standard ones. Customization is very important at this stage, so that small changes (such as colors, logos, images, resources) and much more significant changes (such as process changes, removing or adding modules) can be made to adapt to the reality of each institution. This is known because the system is implemented in a school where such barrier is observed. The system is currently being tested in two educational institutions: the university where the system was developed (UPFIM) and the Tecnológico de Monterrey, Campus Hidalgo. It appears that the system, implemented in a mobile system as it is a widely used tool in that university, efficiently solves specific problems.

The university has informed us of their intention to implement the system in a school on a trial basis.

\section{Analysis of apps for smart mobile devices aimed at supporting academic reinforcement at DES DACI}

Poor student performance is a problem that concerns teachers, parents and educational institutions; it is believed that it could be due to poor study habits, family problems, lack of motivation, among other. The excessive use of smartphones is another noteworthy and current factor related to this problem, as students are more focused on their cell phones than on class, and, in the hallways, they are immersed in social networks, games and movies. All these activities distract them, and therefore they do not devote time to academic activities or simply forget about them, affecting their learning. However, what would happen if, instead of being the cause of the problem, the use of smartphones became an asset to improve academic performance? That, coupled with the use of applications, would benefit the educational environment (Herrera \& Buenabad, 2013).

At present, smartphones are the most used phones by the student community, given the ever-increasing technological demand. At the Dependencia Académica Ciencias de la Información (DES DACl) of Universidad Autónoma del Carmen (UNACAR), located in Ciudad del Carmen, Campeche, Mexico, there is a significant percentage of students with poor academic performance; for that reason, following a quantitative study, it was determined that the university should opt for technological tools to capture the attention of students. After implementing
Evernote, Dropbox and My Homework technologies in the university, the results were encouraging; thus, the university took a first step towards the systematization of its classrooms and teaching processes and, at the same time, it reinforced the idea of having a mobile platform. Introducing these technologies into the education sector would not only provide uniformity to each institution's processes, but would also enhance the academic development of each student by allowing the retrieval of interesting data useful for completing activities such as homework assignments. It is exciting to imagine what would happen if instead of papers, students would use MS Word on their cell phones or save their files in Dropbox and no longer in a folder.

\section{Teachers and the integration of mobile phones in the classroom}

Information and communication technologies in the educational sector must be approached from the perspectives of those involved in each educational activity; to this is added the reaction of teachers, who present the course contents and the annual academic plan and play a fundamental role in the inclusion of these technologies in the classroom. Brazuelo et al. (2017) conducted a study involving teachers of compulsory secondary education in Las Palmas (Canary Islands, Spain) that analyzed the use of smartphones, as well as teachers' attitudes towards the integration of these in academic processes. A certain degree of distrust was observed on the part of teachers towards the use of smartphones in the classroom and the integration of these technologies in academic processes that bring students closer and optimize their work as educators. This situation is more common among older teachers, who are unaware of the benefits that these tools could offer them and the institution. The challenge is to convince them that the tool is functional and is a facilitator of processes to create a better classroom experience.

The age range of the teachers who participated in the study was between 36 and 51 years, and the results were diverse. On the one hand, a group of teachers considered that technology is advancing at a rapid pace; they also stated that the students' experience with devices compared to their lack of training in this area and their age is a factor against them. On the other hand, another group of considerably younger teachers regards smartphones as tools that make communication between parents, students and other members easier, faster and more dynamic. Based on this assessment, we intend to carry out a search for information that serves as a starting point for future research and improvement 
proposals, thus taking a step towards the systematization of educational processes and benefiting society using ICTs for school education on all levels.

\section{Is it possible to integrate mobile devices in ele- mentary education as an educational resource?}

The integration of technological tools and resources in the classroom is a viable reality that facilitates communication between parents, teachers and students. As for the use of mobile resources, it is worth mentioning that their implementation alone will not lead to a significant development of cognitive skills given the current academic situation, a proper teaching planning, where the interrelation of activities is beneficial for students, is essential (Gértrudix \& Barroso, 2016).

A research based on a survey of 68 students in the 5th grade of primary education in a public school located in the province of Toledo, Spain, (Gértrudix \& Barroso, 2016) addresses key aspects such as the number of children with access to a mobile device, usage time and type of applications of their preference. Upon observing the results, it was concluded that children aged 10 and 11 years had mobile devices (smartphones and tablets), a surprising fact given their young age; it was also determined that the most used applications were those for games and messaging, likewise, the number of hours they spent on the mobile phone is also striking. Based on these results, an application for learning historical content in the context of a 5th grade classroom was proposed. The data obtained indicate that elementary school students have different needs and have exchanged toys for technology; parents need to remain vigilant against the excessive use of mobile devices. In some cases, students are only allowed to use their phones on weekends. Teachers should motivate young students to use technology in the best way possible, so that they do not find it boring and do not misuse it.

\section{METHODOLOGY}

First, materials related to the topic, found in indexed databases of recent years, are collected and reviewed in order to know the current status of the research. Then, the analysis and classification of the scientific material directly related to the use of mobile applications in the education sector is performed. Following these instructions, it was possible to select the articles referred to in this research.

Once the relevant material and studies on mobile applications used in education are available, the analysis and description of the model should be conducted before starting to develop the application. The purpose of this analysis is to have a plan to follow to try and optimize communication and interaction between the members of the educational institutions via a mobile application.

Based on the model, the application is developed within a reasonable timeframe using the chosen tools; specific roles (teachers, parents, students and administrative staff) as well as specific functions for each role (tasks, notes, messages, attendance, agenda, news, etc.) are established. Upon completion of the development stage, the mobile application is tested in selected institutions to assess its adaptability to different scenarios for the benefit of education.

\section{RESULTS}

It can be said that academic activities in secondary and higher education concern students and teachers, while at elementary level, it concerns parents, teachers and students; therefore, interaction is somewhat limited (Fuentes, 2015). There is a need to know the current situation in depth and aim at automation.

The proposed system aims to clearly define the users and include administrative personnel, education assistants and all people who are part of the educational environment.

This mobile application conforms to the requirements based on the data collected from the research, which can be summarized as connectivity, usability, user-friendly interfaces, portability, simplicity and optimal complexity.

This mobile application is a tool to transform classroom lessons and modify the way members of each institution interact with each other, promoting constant communication every minute of the day to optimize academic processes and benefit all people involved.

Some parents have old-fashioned ideas and are wary of their youngest children using mobile devices, perceiving them as a distraction. The challenge is to convince parents that the mobile application is in the middle ground between usability and trust (Pinos et al., 2018).

On the other hand, teachers interact with other members of their educational institutions more actively; they already have a routine marked by their experience. This system would restructure academic processes and, via the mobile application, would 
make teachers" work easier; however, based on statistical data, teachers are often reluctant to use ICTs as they are not conversant with them. They need to be trained in the use of mobile devices as they are well aware of the tremendous potential they have if used in the right way.

In addition to all of the above, administrative staff would be able to interact with parents, teachers, students, teacher assistants and mentors through a platform allowing two-way communication; this would encourage the identification of members with the institution to which they belong, as well as trust.

Our proposal is a mobile application that allows to maintain communication and interaction among its users; to send news, events, tasks, attendance records, messages and incidents; to share images of moments lived in the classroom and/or school; and to schedule each deliverable in an orderly manner so that nothing is left to chance. This is an attempt to add value to everything that happens in the classroom with an application that provides a 24-hour connection through notifications.

\section{Conceptualization}

This contribution is intended to analyze the functionality of the system, illustrate its architecture, and explain end-user interaction with the mobile application. It is important to understand how data will be shared among the different users, as the interaction will occur via mobile phones and the workload will be concentrated there. This mobile interaction attempts to be at the forefront in a world where smartphones play an excessive role almost every moment of the day; if they were used for educational purposes to improve performance, a significant advance would be achieved in the educational environment and also in the activities that influence and depend on it.

An educational revolution entails more than just changing a system, it also entails changing the school model from its roots. The present educational model barely includes current technologies; there are technologies that aim to systematize school agendas, but they cannot be properly implemented because the people who make up each part of the model lack the necessary knowledge. The proposed system aims to bridge the existing technological gap in the educational field, as well as the gap that teachers or parents themselves have created as a result of the lack of knowledge on technology.

This system is envisioned as a tool to change the current educational model; however, the system will not be able to accomplish everything by itself, it will be necessary for each entity to be aware of a digital transformation that will bring about this great change.

\section{Target users}

Upon determining the purpose of the system, it is necessary to identify the target audience and their interests, for the needs of a teacher are different from those of a student.

Four types of users were identified: students, parents, teachers, and administrative personnel; each of them responds to a different need, so the most critical functions were identified. The functions that each type of user performs towards interaction and communication between people in each educational institution are shown; furthermore, it is indicated how they perform them now and how they will perform them with the implementation of the system in their study center.

\section{Architectural Model}

As shown in Figure 1, the model identifies components that are of vital importance for the correct functioning of the application; they support the architecture, which is expected to grow over time, as the data and workflow will become increasingly complex due to the number of users interacting with the system in real time.

\section{Database}

The database is important because it contains all the information required for the system to function. The server will be in the cloud so that connections can be made from anywhere, at any time. A good database design will allow the server to remain operational around the clock and, in addition, to be scalable, a very relevant aspect for this system.

The database will be in MySQL format and will have standardized tables to support all the logic to be implemented.

Initially, data will be hosted in the Hostinger server, where the database will be connected and running; from there, all users will be able to connect to it using APIs.

\section{Cloud Messaging Firebase}

This part of the architecture (see Figure 2) relies on the technology provided by Firebase. One of the objectives of this system is that parents, students and teachers are always informed of activities, 


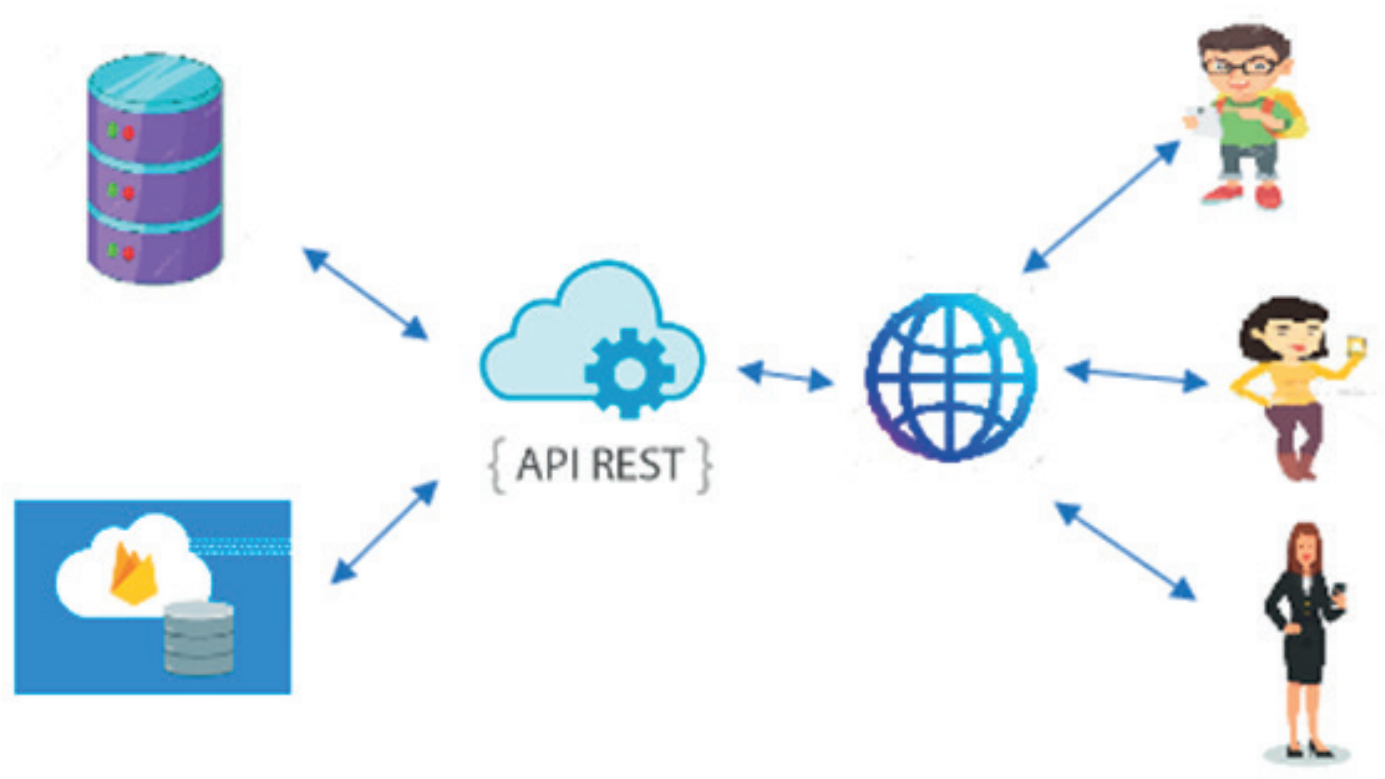

Figure 1. Architectural Model.

Source: Prepared by the authors.

homework or any issue that occurs in the school, to avoid the typical excuses such as "I didn't read the note in my notebook" or "I forgot to tell my mom". In order to manage notifications efficiently, users will rely on Cloud Messaging Firebase.

It provides the following tools for the proper functioning of the system:

\section{- Registration Token}

A registration token request is sent from the mobile device, which enables the mobile device to be identified from any point. Each mobile device has a registration token that is unique in the world, this allows sending personalized messages or notifications that can be mapped.

\section{- Topic Subscription}

Another tool is topic-based subscription, which works in a similar way as when a user subscribes to a YouTube channel to receive notifications. For this educational application, this tool is very important as it allows the user to receive notifications of tasks, events, memos, group chats, or any other data that has been entered into the system. This section is fundamental, since it is where the whole concept of notifying each user in real time is supported by simply sending an unlimited number of notifications on a specified topic from a web service.

For instance, when the teacher enters an assignment with the subject "AssignmentsSchedule5" all students and parents who are enrolled in the schedule with identifier 5 will be notified.

\section{- FirebaseMessagingService:}

- This service, which will be running in the background on mobile devices, it will respond to each notification and will redirect the user to a specific screen by touching each notification. The proper functioning of this service is crucial.

In short, Firebase will enable full control of notifications and will make the project scalable as these services support a large amount of data.

\section{API REST}

It is necessary to develop the APIs for an efficient communication between the mobile devices and the server. These APIs are developed in PHP and java, which makes their maintenance and updates processes easier. The logic of the model interacts directly with the database, retrieving data when requested through JSON, which is then returned to be processed in Android. 


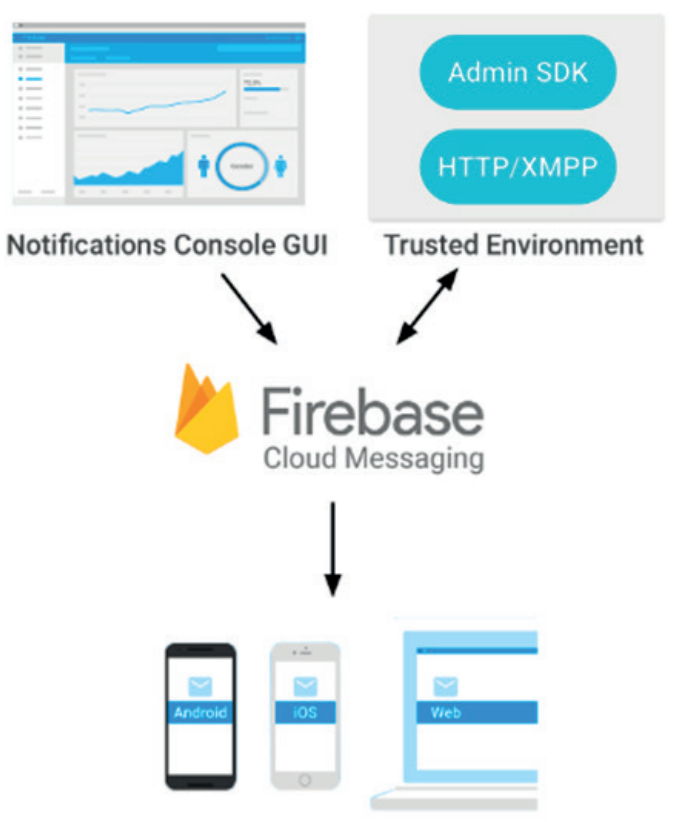

Figure 2. Cloud Messaging Firebase.Source. Source: Prepared by the authors.

These APIs also call the services provided by firebase doing all the logic from here. The connection and the transformation to JSON. Proper use of this part of the architecture ensures proper operation and efficient maintenance.

\section{Internet}

As any current system must have internet coverage, the connection is the most important thing to send notifications or post any event. Efforts are made to consume as few megabytes as possible, and to send as few requests to the server as possible using HTTP.

Efficient use of this resource provides an optimal solution for the user.

\section{End-users}

It is at this stage of the architecture when the mobile application becomes important, users interact with the mobile application to, in turn, interact with other users associated with each educational institution. Teachers will be in charge of subjects and students who in turn will be connected to parents.

All mobile devices will be connected to each other and it will be easy to collect data within each module. The architecture is designed so that the user can locally access the information stored in the local database, even if he/she has run out of data. In this case, however, the user will have access only to previously viewed information.

This information will be useful for users because they will be able to access their tasks, notes, events and even messages without internet access, thus making the architecture more robust.

\section{Surveys}

In order to implement this system, we gathered information about the target market, i.e., elementary and secondary schools. Surveys were used for this purpose, as they allow obtaining reliable data to ensure a solid basis for our project (Sanchez et al., 2014). The surveys were conducted in Cono Norte in three stages: the first was carried out prior to product launching; the second, days after launching the product; and the third, after the end of the first school term.

\section{- Prior to product launching}

The purpose of this part of the survey was to assess how students, parents, teachers, principals and all the people involved in the educational institutions would receive a mobile application that is intended to improve the interaction between them.

At pre-school level, it was observed that a student module was not so necessary, given that only 3 students (5 years old) 
had smartphones, whereas all the teachers had one. Also, it was observed from the survey that both parents and teachers at this level are at the forefront of technology. At elementary level, it was observed that a small percentage of teachers did not have a smartphone, whereas a large number of students had one. At this level, students had restricted data usage and, those who did not have data, stated that they accessed the Internet at home. At secondary level, it was noted that teachers, who were older, often had basic mobile phones rather than smartphones, whereas almost all students had one; in addition, teachers often did not have access to data, but were willing to acquire a data plan.

It was also observed that the limited knowledge regarding educational platforms constitutes a barrier hindering the transformation to the digital era. It is mainly parents and students who have little or no knowledge on the use of such platforms, while teachers or administrative staff do have experience in this regard due to the use of virtual classrooms during their training or because they have seen some on the Internet. For students who do not have access to a mobile phone, the best form of communication is through Facebook; while, for those who do have one, communication through WhatsApp is better.

The interest that people showed in the possibility of having a system that notifies them of something as simple as homework was quite surprising; this response suggests that they see the system as a potential solution to a need. Users do not want a new e-mail and password, they stated that it would be better to use a system similar to WhatsApp, where users only need to register once and that, when logging into the system, they can log in directly, which makes the process easier.

The results obtained in the first surveys are encouraging. In accordance with what has been stated, we want to provide a system that satisfies the basic needs of parents, students and teachers in order to contribute to academic development; at the same time, it is important to recognize that this is not only an application, but a change in the learning and communications model.

\section{- Days after product launching}

According to the results, the system was well received by the users and the people surveyed were duly informed by the schools about the use of the application in favor of the digital transformation. User acceptance rate was above $90 \%$, which shows that the system is on the right track. Likewise, a $92 \%$ acceptance rate was observed from teachers, something that was surprising at the time, but that would be useful to identify problems or issues that may not have been evident. Students, parents and administrative personnel were satisfied, since their function fully complies with the parameters that were projected. A 100\% approval rate was obtained in terms of usability, and simplicity of the screens.

The system had to be tested to verify if notifications were being delivered in real-time every time there was an interaction; it was very important since we depended on the Firebase service. It was gratifying to know that users were being notified correctly, which proved the usability and functionality of the system.

- At the end of the first school term (August 26, 2018)

This third stage of the survey was directed to the teachers, since, after closing the first period (bi-monthly or quarterly), it was necessary to know if the system had worked as expected and if the users' reaction had been satisfactory. To this end, a 5-question survey was prepared for 50 teachers from the different schools we approached. Results showed that all teachers agreed that the homework module had been very useful. They even felt that the system made their job easier, since they were able to send homework to all their students and parents in a matter of seconds. Therefore, sending time was reduced and communication was maintained.

It was also noted that the grading module was very useful for teachers because it fulfilled the function of communicating. It is not a super module for calculating averages, but a record of grades; the teachers were able to understand it and it was useful, however, the system still needs to be optimized and transformed into a virtual classroom. 
Teachers were happy with the system, and while it has potential, there are still areas for improvement. This is the first version of the system and it complies with its function, makes the users happy and, therefore, the first stone for the digital transformation has been laid.

The surveys were conducted in three different stages, thus providing a great insight into what the system is capable of delivering in the educational institutions. The digital transformation can be observed using these data.

\section{Application Development}

\section{Interfaces}

In order to better visualize the proposed system, the interfaces designed for the school interaction and communication system, which we have called MottyApp, are presented.

Figures 3 and 4 show the screen that the user will see after downloading the application from Play Store. The user code that will be provided when purchasing the system is entered on this screen. Once the code is entered, the system will associate the user with the code and will display Figure 5.

Figures 6 and 7 represent the main screens according to the user assigned. Each user has its own modules, some of which differ in terms of functionality, while others are similar. The sidebar was designed for the user's convenience, since it is much easier to have all the options on the main screen, thus avoiding unnecessary system flow.

Figure 8 represents the news display screen, which will slide upwards, resembling Facebook, to make the user feel comfortable and more familiar with the application.

Figure 9 shows the album module that will display the albums created by users in an organized manner with the tag "new", so that the network is updated on the number of photos and albums. Each album will be associated to a subject or section, to which only authorized persons will have access; there is no limit to the number of photos that can be added to the albums.

Figure 10 represents the schedule screen, one of the most important screens of the system, for it will

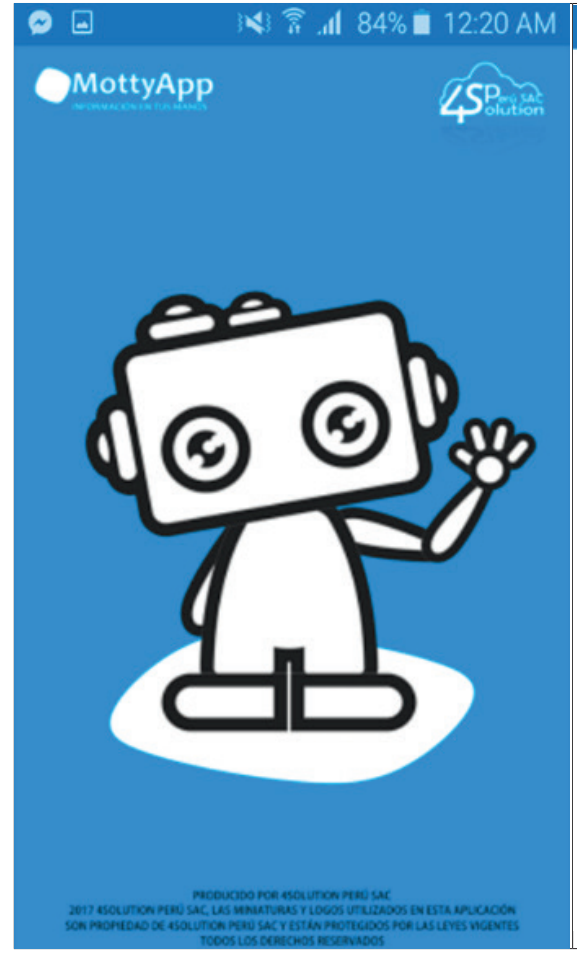

Figure 3. Startup splash screen. Source: Prepared by the authors.

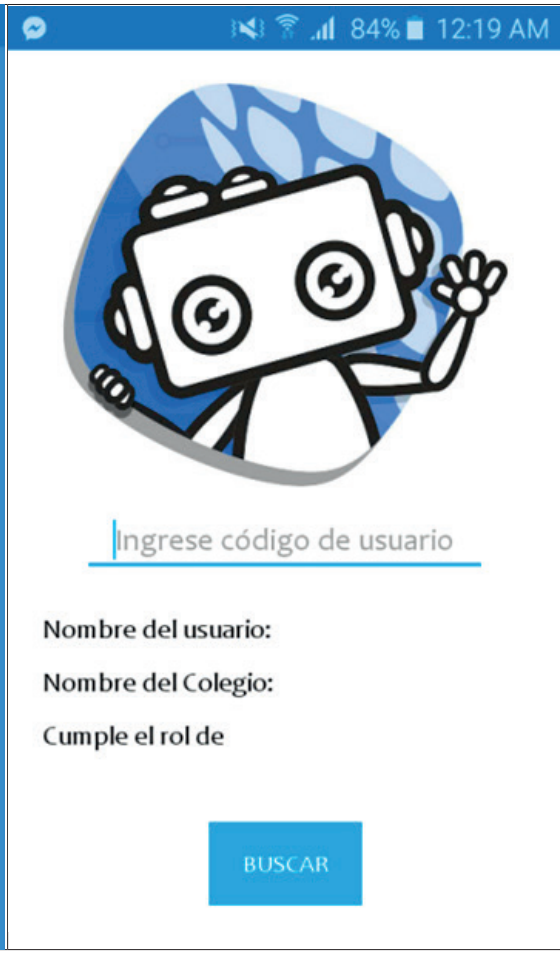

Figure 4. Registration screen. Source: Prepared by the authors.

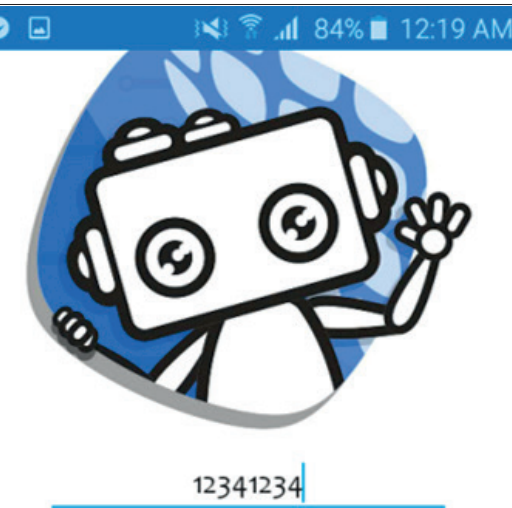

Nombre del usuario: Torres Davila George Deave

Nombre del Colegio: Jean Paul Sartre Cumple el rol de profesor

Figure 5. Registration confirmation screen.

Source: Prepared by the authors. 


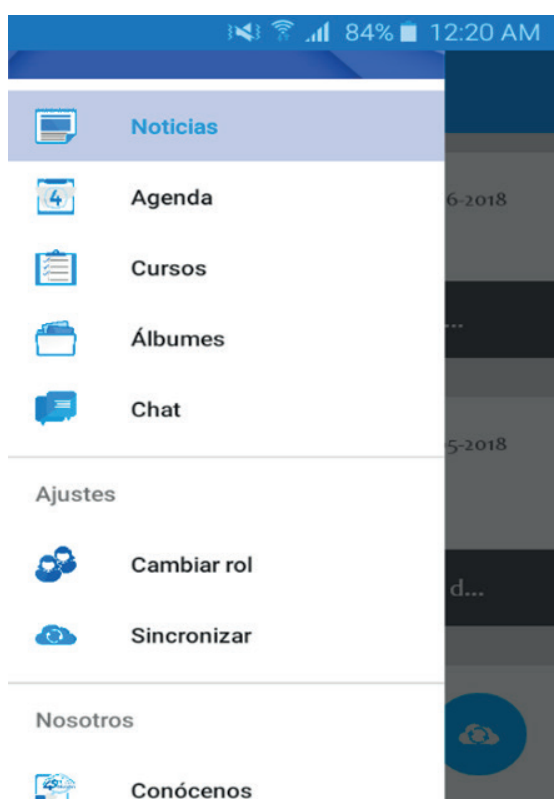

Figure 6. Main screen (student). Source: Prepared by the authors.

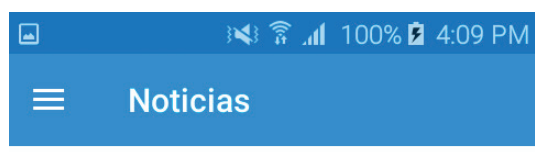

C Colegio_Prueba

14-11-2017

Reconocimiento de mejores proyectos

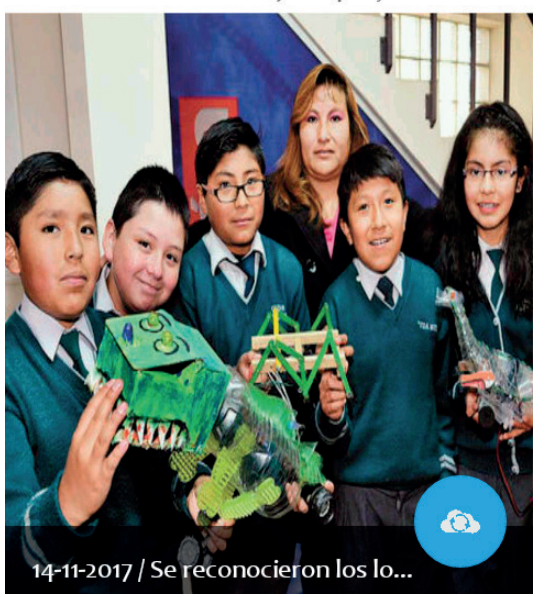

Figure 8. News display screen. Source: Prepared by the authors.

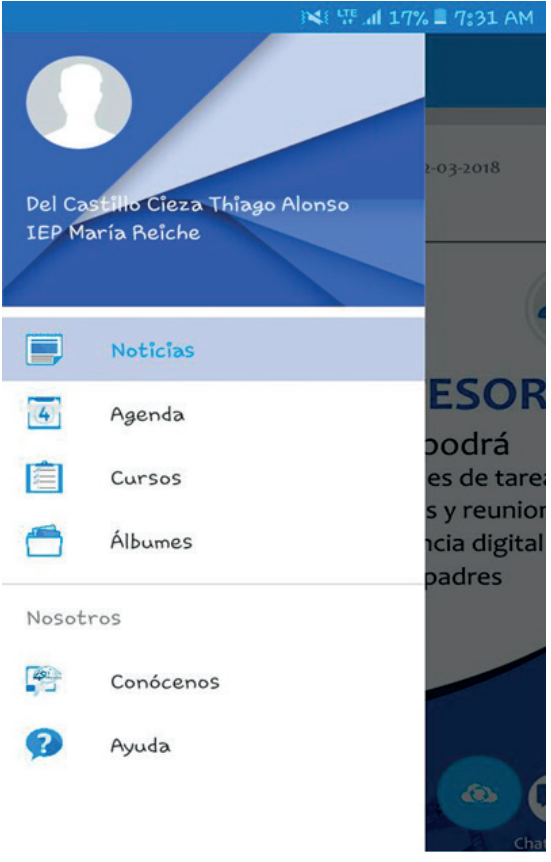

Figure 7. Main screen (teacher). Source: Prepared by the authors.
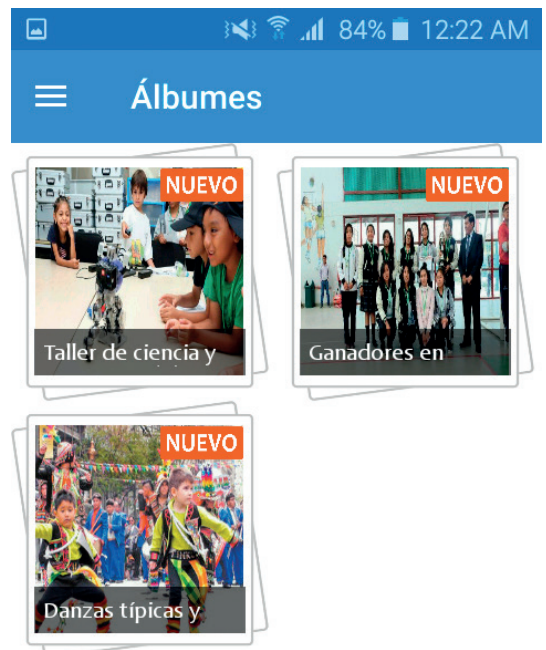

Figure 9. Album screen. Source: Prepared by the authors. 
collect all the information to be displayed in a calendar using different colors with their own meanings to indicate whether it is a task, an event, a meeting, etc.

To see what is scheduled for each day, you only need a touch to see the pending tasks for that day organized on a single screen.

Figure 11 represents the subject screen that will list the courses in alphabetical order and associated with an image to make browsing easier for the user.

The images displayed are dynamic and change according to the season of the year. These courses are the ones that the student takes; if you are a parent, they are those taken by ones your child =; and, if you are a teacher, they are the courses that you teach. Images are used to organize and systematize the information.

Figures 12 and 13 represent the menus within each course and have well defined functionalities. It is a very interactive menu and full of colors to attract people's attention; each course will have its own menu of tasks, incidences, events, attendances, etc., to maintain order. This menu slides upwards so that the user finds it familiar, and the transition to this tool is easy.

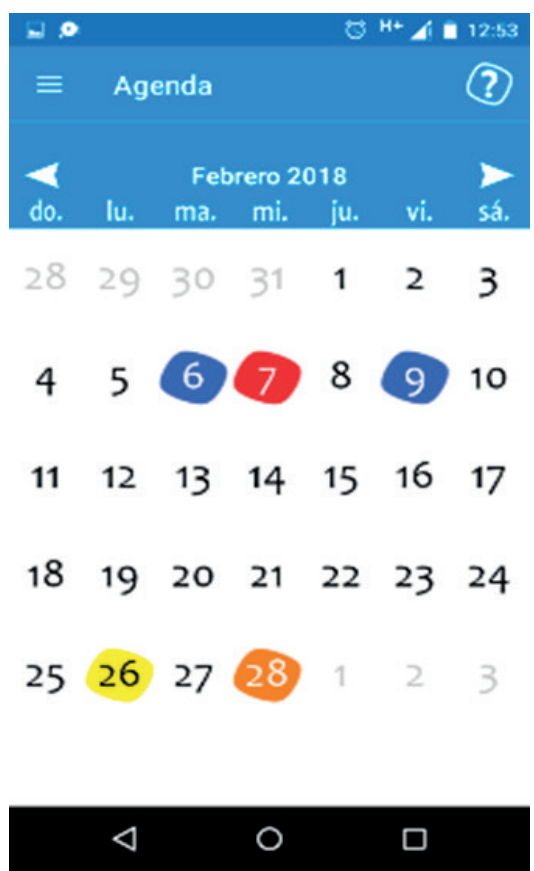

Figure 10. Schedule screen. Source: Prepared by the authors.
Figures 14, 15, 16 present some of the screens that will be seen within the course menu, all following the same design to maintain uniformity, which represents exactly our proposal: an organized, useful and user-friendly system.

Figures 17 and 18 represent the parents and teachers' module, as well as the chat, which will contribute to the solution of the communication and interaction problem by bridging the existing gap; its design is user-friendly and dynamic. Figure 19 represents the parents' GPS module, aimed at bringing them closer to the students, since parents will know the exact location of their child in real time just by entering the module, allowing them to monitor their children.

\section{DISCUSSION}

Upon initiating this project, we noticed that some schools used messaging applications to perform their activities and maintain communication with the participants of the classrooms.

Using the applications available in the market to perform school activities makes browsing and receiving pending messages tedious, order cannot be maintained and not all people are familiar with these apps. Therefore, this study seeks to maintain

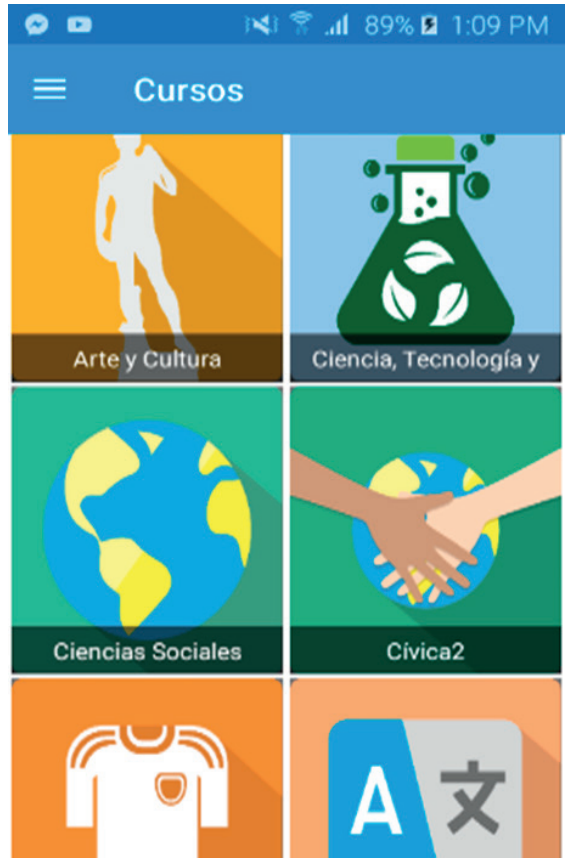

Figure 11. Subject screen. Source: Prepared by the authors. 


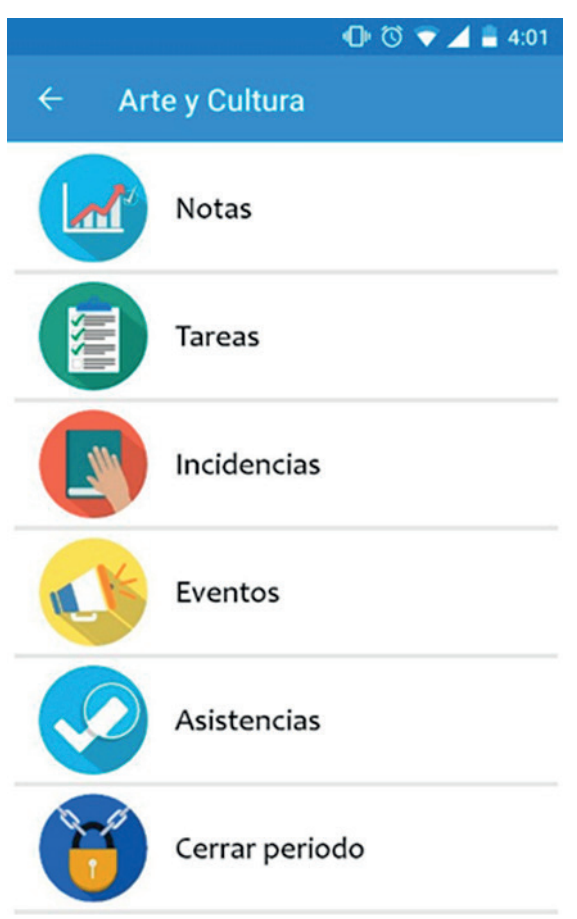

Figure 12. Courses menu (teacher). Source: Prepared by the authors.

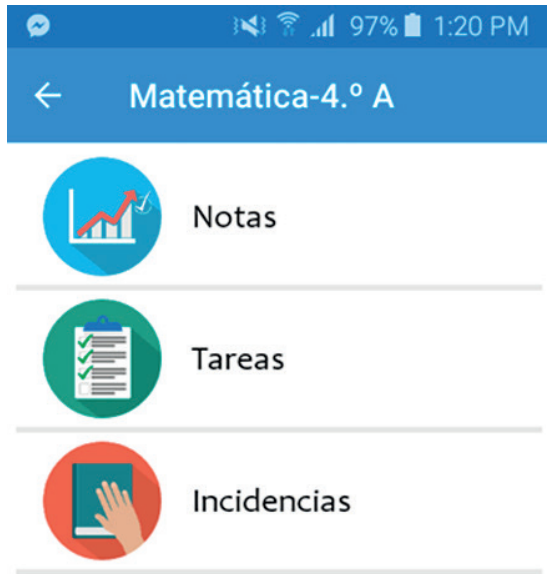

\section{Eventos}

Asistencias

Figure 13. Course menu (parent and student)

Source: Prepared by the authors.

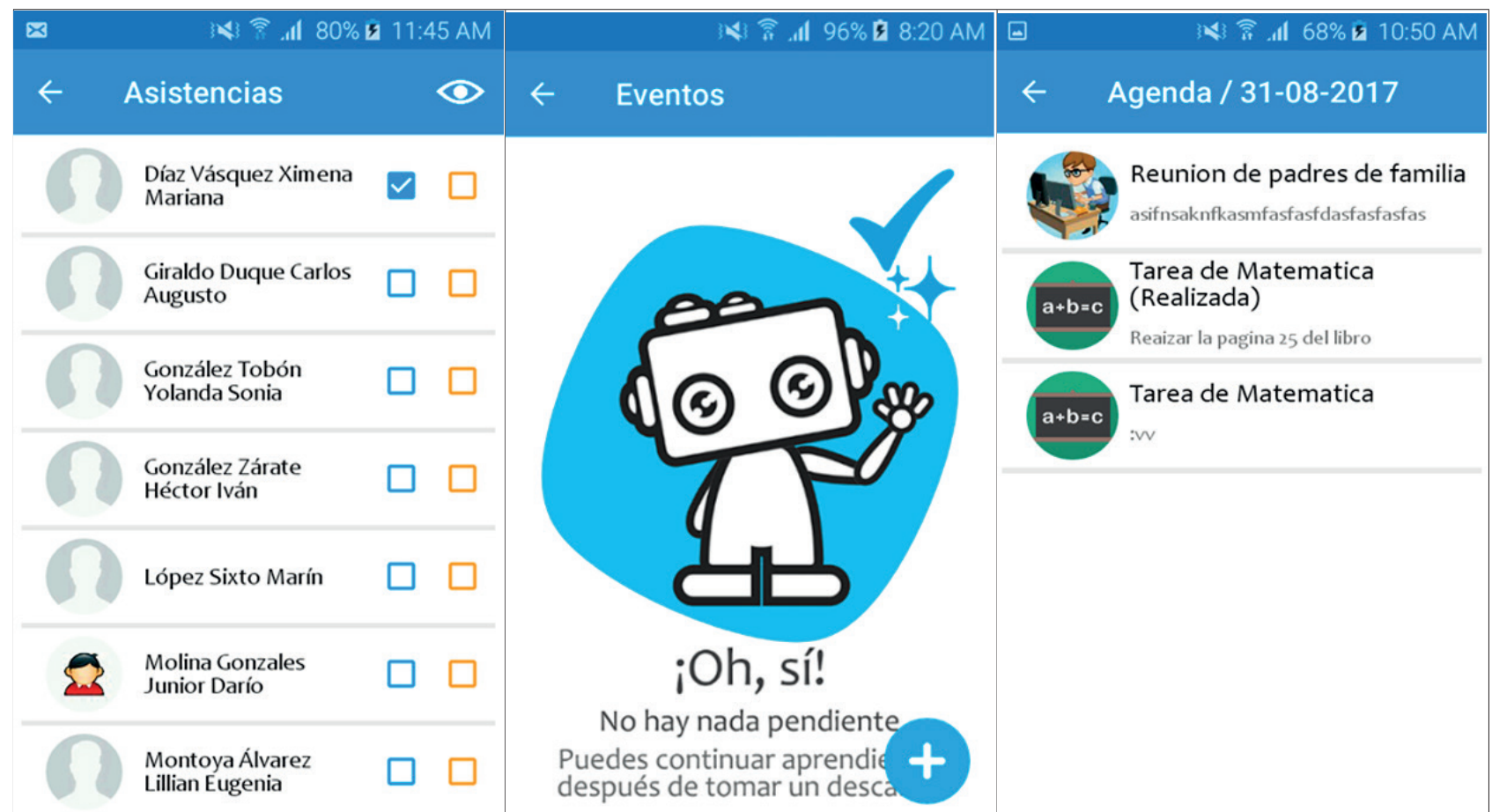

Figure 14. Attendance.

Source: Prepared by the authors.
Figure 15. Events.

Source: Prepared by the authors.
Figure 16. List of pending tasks. Source: Prepared by the authors. 


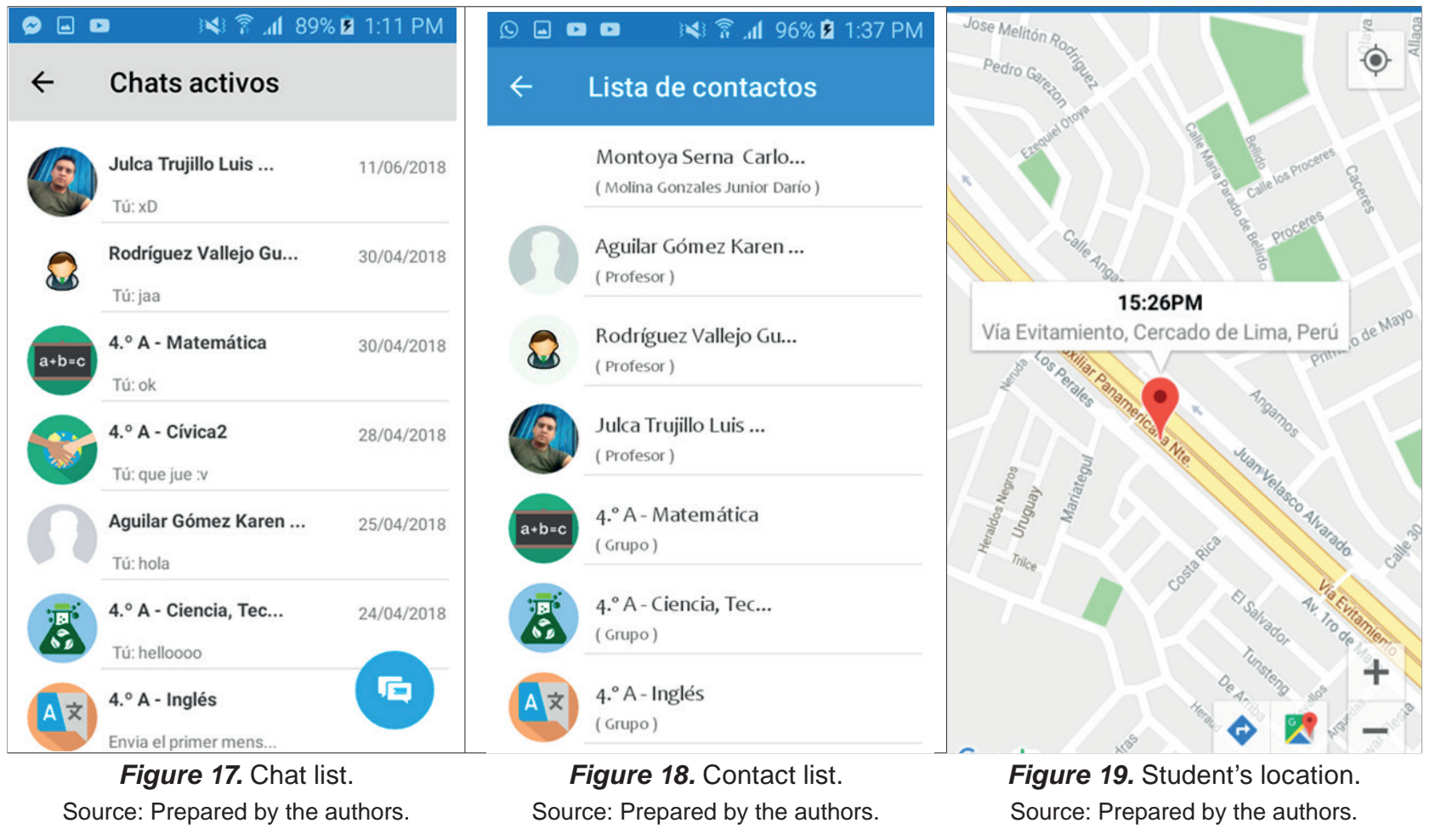

an order for each activity, whether it is a to-do list, notes, homework, memos, messages, etc., via an intuitive, effective, and easy-to-use application that can be used by people of any age. In this sense, this application attempts to take a step forward in the use of technology in schools in order to contribute to the academic growth of students, as well as to the comfort of teachers and parents.

\section{CONCLUSIONS}

It is clear from the study conducted that there is a gap between the educational sector and communication. In a world of technology like ours, it is important to take advantage of the technology that we have at hand every day, i.e., smartphones.

Communication and interaction between the people involved in an educational institution is deficient; however, the system we propose is a big step towards improvement and responds to a need that, in general, does not receive the necessary attention. Thanks to the information provided before the implementation of the mobile application and to the people surveyed and informed about the benefits of this system, together with the fact that they have functionally interacted with it, it can be said that the system provides solutions to the long-standing issue of communication between parent-student-teacher.
This mobile application has its limitations, but it is the starting point towards digital transformation. The modules were developed bearing in mind the needs of the people involved. It provides a simple way to address those needs and allows for the possibility of improving the modules, as well as recognizing new functionalities that may arise in the future. The system complies with the stipulated in the problem statement and the study was formally conducted.

\section{REFERENCES}

[1] Arias, W. (2015). Tecnologías de la información y la comunicación en colegios públicos y privados de Arequipa. Interacciones, 1(1), 1128.

[2] Brazuelo, F., Gallego, D., \& Cacheiro, M. (2017). Los docentes ante la integración educativa del teléfono móvil en el aula. RED. Revista de Educación a Distancia, 52(6). Retrieved from http://dx.doi.org/10.6018/red/52/6

[3] Cataldi, Z., Méndez, P., Dominighini, C., \& Lage, F. (2012). Dispositivos móviles en educación superior y entornos personalizados de aprendizaje. XIV Workshop de investigadores en ciencias de la computación, 1014-1018.

[4] Díaz, J.., Ucán, J., Aguileta, A., \& Toscano, A. (2016). Asistente escolar para los estudiantes 
de Ingeniería de Software: una aplicación móvil. ReCIBE, 5(3).

[5] Fuentes, O. (2015). La organización escolar. Fundamentos e importancia para la dirección en la educación. VARONA, 61, 1-12.

[6] Gértrudix, F., \& Barroso, M. (2016).¿Es posible integrar los dispositivos móviles en educación primaria como recurso educativo? In Gómez, J., López, E., \& Martín, A. (Eds.), Advances and Innovations in Educational Research (pp. 35-43). San Juan, Puerto Rico: UMET Press and editors.

[7] Gomez, M., García, E., \& Reyna, O. (2016). Aplicación Móvil para Control Escolar. Revista de Aplicación Científica y Técnica, 2(4), 1-5.

[8] Herrera, B., \& Buenabad, M. (2013). Análisis de las aplicaciones para dispositivos móviles inteligentes en apoyo al fortalecimiento académico en la DES Ciencias de la Información. Revista Iberoamericana Para La Investigación y El Desarrollo Educativo, 11.

[9] Mendoza, M. (2014). El teléfono celular como mediador en el proceso de enseñanzaaprendizaje. Omnia, 20(3), 9-22.

[10] Molina, A., Roque, L., Garcés, B., Rojas, Y., Dulzaides, M., \& Ganén, M. (2015). El proceso de comunicación mediado por las tecnologías de la información. Ventajas y desventajas en diferentes esferas de la vida social. Medisur, 13(4).
[11] Muñoz, E., Fernández, D., Gómez, J., \& Arevalo, C. A. (August 13-15,2015). Impacto de Aplicaciones para Dispositivos Móviles: Un estudio de Aceptación [scientific article]. Twenty-First Americas Conference on Information Systems, San Juan, Puerto Rico.

[12] Paredes-Parada, W. (2019). Brecha en el uso de tecnologías de la información y comunicación (TIC) básicas y modernas entre estudiantes y docentes en universidades ecuatorianas. Revista Educación, 43(1). Retrieved from https://doi.org/10.15517/revedu.v43i1.27423

[13] Pinos, N., Hurtado, S. \& Rebolledo, D. (2018). Uso del teléfono celular como distractor del proceso enseñanza - aprendizaje. Enfermería Investiga, Investigación, Vinculación, Docencia y Gestión, 3(4).

[14] Sánchez, B., Diez, G., \& Buenabad, M. (2014). El uso de los teléfonos móviles, las aplicaciones y su rendimiento académico en los alumnos de la DES DACI. Revista Iberoamericana Para La Investigación y El Desarrollo Educativo, 12.

[15] Villalonga C., \& Marta-Lazo, C. (2015). Modelo de integración educomunicativa de 'apps' móviles para la enseñanza y aprendizaje. Pixel-Bit Revista de Medios y Educación. (46), 137-153. 
